九州大学学術情報リポジトリ

Kyushu University Institutional Repository

\title{
GENERATIVE AND GENEALOGICAL CLASSIFICATIONS OF ALL THE CONFIGURATIONS IN AN \$ M TIMES N \$ CELL SPACE UNDER APPLICATIONS LOCAL MAJORITY TRANSFORMATION : INFORMATION SCIENCE APPROACH TO BIOMATHEMATICS, XIII
}

Kitagawa, Toshio

Research Institute of the Fundamental Information Science, Faculty of Science, Kyushu University

https://doi.org/10.5109/13065

出版情報: 統計数理研究. 15 (1/2)，pp. 85-112，1972-03. Research Association of Statistical Sciences バージョン：

権利関係 : 


\title{
GENERATIVE AND GENEALOGICAL CLASSIFICATIONS OF ALL THE CONFIGURATIONS IN AN $m \times n$ CELL SPACE UNDER APPLICATIONS OF LOCAL MAJORITY TRANSFORMATION \\ -INFORMATION SCIENCE APPROACH TO BIOMATHEMATICS, XIII
}

\author{
By \\ Tosio Kitagawa* \\ (Received November 13, 1971)
}

\section{Introduction and fundamental notions}

The object of this paper is to give two kinds of classifications, called generative and genealogical respectively, of all the configurations in an $m \times n$ cell space with unit square cells, which are subject to all the possible applications of local majority transformation. This paper is a continuation of our previous papers [1], [3] and Yamaguchi [2] and several definitions regarding fundamental notions such as unit cells, $2 \times 2$ basic spaces, local majority transformations and applications of firing points introduced in these previous papers of ours will be also used in this paper without any further specification.

In particular a configuration $C$ in an $m \times n$ cell space is said to be stable, if for any assigned configuration $C^{\prime}$, which is different from $C$, the probability of occurrance of the central firing points which induces a mapping transformation from the configuration $C$ to $C^{\prime}$ is equal to zero. In our previous paper [1], we show that in an $m \times n$ cell space the number of all the possible stable configurations is $2^{m+n-1}$. (Theorem in [1]). These $2^{m * n-1}$ stable configurations play the fundamental role in our classification of all the possible $2^{m n}$ configurations in an $m \times n$ cell space. The main result of this paper regarding a generative classification of all configurations is given by

THEOREM I. Let us denote by $K(m, n)$ the set of all the possible configurations in an $m \times n$ cell space. Then we have

$$
K(m, n)=\sum_{i=1}^{\varphi(m, n)} K^{(i)}(m, n),
$$

where each $K^{(i)}(m, n)\left(i=1,2, \cdots, \varphi(m, n) \equiv \varphi_{m, n} \equiv 2^{(m-1)(n-1)}\right)$ is a set of certain $2^{m+n-1}$ configurations in an $m \times n$ cell space such that, for $i \neq j$,

* Research Institute of the Fundamental Information Science, Faculty of Science, Kyushu University. 


$$
K^{(i)}(m, n) \cdot K^{(j)}(m, n)=\phi .
$$

Furthermore each $K^{(i)}(m, n)$ is the $i$-th class, where the 1 -st class is called an Eden generation, while the $\varphi(m, n)$-th class is equivalent to the set of all the stable configurations in the $m \times n$ cell space.

The meaning of an Eden generation comes from the well known definition of Eden garden currently used in automation theory. In fact we appeal to

Definition 1.1. A configuration $C$ in an $m \times n$ cell space is said to belong to an Eden generation, if satisfies the following two conditions:

$\left(1^{\circ}\right)$ There is no other configuration from which the configuration $C$ can be obtained by an application of LMT.

$\left(2^{\circ}\right)$ By any application of LMT, the configuration $C$ changes to some other configuration different from $C$.

Thus an Eden generation and the set of all the stable configurations are the two extreme types of configurations between which there exists a set of all other configurations belonging to the classes of the 2 nd to the $\left(2^{(m-1)(n-1)}-1\right)$-th.

The classification is due to a systematic scheme of applications of $\Delta$-operations, which will be defined in Section 2, to the set of all the stable configurations in an $m \times n$ cell space, that is, $K_{\varphi(m, n)}(m, n)$. In Section 3 we shall give the proof of THEOREM I on the basis of our three Theorems given in our previous paper [1] and by means of $\Delta$-operations. This proof itself gives us a systematic construction procedure of all the possible configurations in an $m \times n$ cell space, by which we can obtain each individual configuration without detailed consideration on the situations from which it has come and to which it will go, by all the possible applications of local majority transformation (LMT), that is to say, without genealogical considerations.

In this sense the classification of all configurations is called to be generative.

Section 4 gives two simplest examples of our THEOREM I by showing the results of the generative classification for $2 \times 2$ and $2 \times 3$ cell spaces respectively.

In order to discuss a genealogical classification, we introduce the following fundamental notions in genealogy of configurations in an $m \times n$ cell space under all the possible applications of LMT.

Definition 1.2. A configuration $B$ is called to be a direct descendant of a configuration $A$ if $A$ and $B$ are different configurations and there is at least one firing point in an $m \times n$ cell space such that the application of LMT at this firing point will transform the configuration $A$ to the configuration $B$.

In this case a configuration $A$ is called to be a direct ancestor.

For each assigned set $n$ of configurations the set of all the direct descendants (ancestors) is denoted by $d(\mathfrak{H})(a(\mathfrak{H}))$. Particularly when $\mathfrak{U}=\{A\}$, let us write simply $d(A)(a(A))$ instead of $d(\{A\})(a(\{A\}))$.

We proceed to introduce

DEFINition 1.3. A configuration $A_{n}$ is called to be a descendant (or ancestor) of $A_{1}$, if there is a finite sequence of configurations $\left\{A_{i}\right\}(i=2,3, \cdots, n-1)$ such that each $A_{i \div 1}$ is a direct descendant (ancestor) of $A_{i}$, that is, $A_{i+1} \in d\left(A_{i}\right)\left(A_{i+1} \in a\left(A_{i}\right)\right)$ for $i=$ 
$1,2,3, \cdots, n-1$.

It is convinient to define a sequence of configurations $\left\{d^{(\nu)}(A)\right\}$ and $\left\{a^{(\nu)}(A)\right\}$ such that

$$
\begin{aligned}
& d^{(0)}(A)=\{A\} \\
& d^{(\nu)}(A)=d\left(d^{(\nu-1)}(A)\right) \quad(\nu=1,2,3, \cdots)
\end{aligned}
$$

and similarly

$$
\begin{aligned}
& a^{(0)}(A)=\{A\} \\
& a^{(\nu)}(A)=a\left(a^{(\nu-1)}(A)\right) \quad(\nu=1,2,3, \cdots) .
\end{aligned}
$$

DEFINITION 1.4. The set of all the descendants (ancestors) of a configuration $A$ is called to be the decendant (ancestor) tree of $A$ and is denoted by $\mathfrak{D}(A)(\mathfrak{H}(A))$.

Due to this definition we have

$$
\begin{aligned}
& \mathfrak{S}(A)=\sum_{v \geqq 0} d^{(\nu)}(A) \\
& \mathfrak{R}(A)=\sum_{v \geqq 0} a^{(\nu)}(A) .
\end{aligned}
$$

For any assigned set of configurations $\mathbb{B}$, let us define

$$
\begin{aligned}
& \mathfrak{A}((\mathbb{S}) \equiv[\mathfrak{A}(B) ; B \in \mathbb{S}] \\
& \mathfrak{D}(\mathbb{G}) \equiv[\mathfrak{D}(B) ; B \in \mathbb{G}] \\
& {[\mathfrak{H}, \mathfrak{D}](\mathbb{B}) \equiv \mathbb{S}+\mathfrak{Y}(\mathbb{B})+\mathfrak{D}(\mathbb{S}) \equiv \mathbb{S}^{(1)}} \\
& \mathbb{S}^{(n)} \equiv[\mathfrak{H}, \mathfrak{D}]^{(n)}(\mathbb{S}) \\
& \equiv[\mathfrak{A}, \mathfrak{D}]\left(\mathbb{S}^{(n-1)}\right) \quad(n \geqq 1) \\
& \mathbb{B}^{(0)} \equiv \mathbb{B} \text {. }
\end{aligned}
$$

Then it follows that

$$
\mathbb{G}^{(0)} \cong \mathbb{G}^{(1)} \cong \mathbb{B}^{(2)} \leqq \cdots .
$$

Because of the finiteness of the set of all the configurations in an $m \times n$ sell space, there is an $n_{0}$ such that

$$
\mathbb{B}^{\left(n_{0}-1\right)} \subsetneq \mathbb{G}^{\left(n_{0}\right)}=\mathbb{B}^{\left(n_{0}+1\right)}=\cdots .
$$

Here let us introduce

DEFINITION 1.5. For each assigned set $\$ \$$ of configurations, the set of all the configurations defined by

$$
\mathfrak{L}(\mathbb{S}) \equiv \lim _{n \rightarrow \infty} \mathbb{B}^{(n)}=\mathbb{S}^{\left(n_{0}\right)}
$$

is called to be the lineage tree of $\mathbb{G}$.

Specially when $\mathbb{S}$ consists of a single configuration $A$, then we shall write $\mathbb{R}(A)$ instead of $\mathfrak{L}(\{A\})$.

Now the problems regarding genealogy of configurations in an $m \times n$ cell space are concerned with:

$\left(1^{\circ}\right)$ To find out $\mathscr{D}(A)$ and $\mathfrak{H}(A)$ with their respective connections under LMT 
for each assigned configuration $A$.

$\left(2^{\circ}\right)$ To find out $\mathfrak{L}(A)$ with its connection under LMT for each assigned configuration $A$.

$\left(3^{\circ}\right)$ To decompose the set of all configurations in an $m \times n$ cell space into a set of mutually disjoint lineages.

In Section 5 we shall give a general procedure to solve these three cell spaces in a systematic way. It is observed that the decomposition of all the configurations in an $m \times n$ cell space into the set of mutually disjoint lineages corresponds to a component decomposition of a graph, and we may and we shall call our second main classification result enunciated in LEMMA 1 given in Section 5 a genealogical classification of all the conggurations in an $m \times n$ cell space under LMT.

An example is given with respect to a $3 \times 3$ cell space. There are several conconsiderations including rotation groups of configurations which make our enumeration work simpler than otherwise we would be involved as shown in these Examples.

Moreover it turns out that in an $m \times n$ cell space all the non-isolated stable configuration belong to the same one lineage, while each individual isolated stable configuration constitutes one lineage respectively, which is singular in the sense that it has no ancestor and no descendent different from itself. THEOREM II shows an existence of connection route of all the configurations in an $m \times n$ cell space except all isolated stable configurations. Because of THEOREM II our notion of lineage may seem to be too broad to describe connection relations among the set of all the configurations in an $m \times n$ cell space. In fact, apart from isolated stable configurations, all the other configurations belong to the same one lineage, which makes LEMMA 1 to be non-substantial. Neverthless for some proper subsets of all the configurations the notion of lineage can be expected to have its existence value.

\section{2. $\Delta$-operation in an $m \times n$ cell space}

Let $a=\left(a_{1}, a_{3}, \cdots, a_{n}\right), b=\left(b_{2}, b_{3}, \cdots, b_{m}\right)$ be any assigned two sets of real numbers where each $a_{i}$ and each $b_{j}$ are either of 1 and 0 .

Let $C(m-1, n-1)$ be any assigned configuration in an $(m-1) \times(n-1)$ cell space such that

$$
C(m-1, n-1)=\left(\begin{array}{cccccc}
x_{22} & x_{23} & \cdots & x_{2 j} & \cdots & x_{2 n} \\
x_{32} & x_{33} & \cdots & x_{3 j} & \cdots & x_{3 n} \\
\cdots \cdots \cdots \cdots \cdots & \cdots \cdots \cdots \\
x_{i 2} & x_{i 3} & \cdots & x_{i j} & \cdots & x_{i n} \\
\cdots \cdots \cdots \cdots \cdots \cdots \cdots & \cdots \\
x_{m 2} x_{m 3} & \cdots & x_{m j} & \cdots & x_{m n}
\end{array}\right)=X .
$$

Now let us introduce

Definition 2.1. A A-operation applied to a configuration $X=C(m-1, n-1)$ with a boundary condition $B d(a, b)$ is defined as a transformation of the configuration $C(m-1, n-1)$ into a configuration in an $m \times n$ cell space $C_{b}^{a}(m, n)$ and is denoted by $\triangle_{b}^{a} X$ such that 


$$
\triangle_{b}^{a} X=\triangle_{b}^{a} C(m-1, n-1)=C_{b}^{a}(m, n),
$$

where we put

and

$$
C_{b}^{a}(m, n)=\left(\begin{array}{cccccc}
a_{1} & b_{2} & \cdots & b_{j} & \cdots & b_{n} \\
a_{2} & y_{22} & \cdots & y_{2 j} & \cdots & y_{2 n} \\
a_{3} & y_{32} & \cdots & y_{3 j} & \cdots & y_{3 n} \\
\cdots \cdots \cdots \cdots \cdots \cdots \cdots & \cdots \cdots \\
a_{i} & y_{i 2} & \cdots & y_{i j} & \cdots & y_{i n} \\
\cdots \cdots \cdots \cdots \cdots \cdots \cdots & \cdots \cdots \\
a_{m} & y_{m 2} & \cdots & y_{m j} & \cdots & y_{m n}
\end{array}\right)
$$

$$
y_{i j} \equiv a_{i}+b_{j}+\sum_{p=2}^{i} \sum_{q=2}^{j} x_{p q} \quad(\bmod .2)
$$

for $i=2, \cdots, m ; j=2, \cdots, n$.

For the sake of brevity, inview of (2.01), we shall write

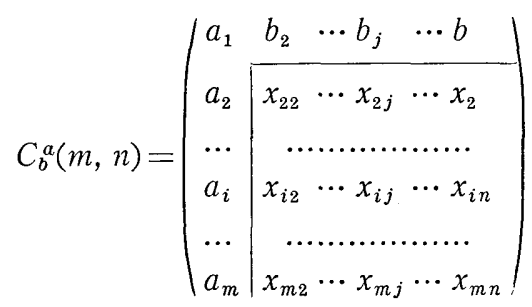

$$
\begin{aligned}
& =\left(\begin{array}{cccc}
a_{1} & b_{2} & \cdots & b_{n} \\
a_{2} & & \\
\vdots & X \\
a_{m} & &
\end{array}\right) \text {, simply. }
\end{aligned}
$$

Now it can be readily seen

COROLLARY 2.1. The set of all the stable configurations in an $m \times n$ cell space can be obtained as the set of all the configurations generated by $\triangle_{b}^{a} X_{0}$ when $a$ and $b$ in a boundary condition $B d(a, b)$ take all the possible combinations with the particular configuration $X_{0}$ which consists of all zero elements.

In fact we have

$$
y_{i, j}=a_{i}+b_{j}, \quad(i=2,3, \cdots, m ; j=2, \cdots, n)
$$

which shows that $C_{b}^{a}(m, n)$ is a stable configuration for each assigned set of two vectors $a$ and $b$ as a boundary condition.

This Corollary yields us another proof of the following two fundamental results given in THEOREMS 2 and 3 in our previous paper [1].

THEOREM 2. A configuration which is complementary to a stable configuration is stable.

THEOREM 3. A stable configuration in an $m \times n$ cell space has the following pattern: 
$\left(1^{\circ}\right)$ There is a pair of partitions such that

$$
\left\{\begin{array}{l}
m=m_{1}+m_{2}+\cdots+m_{k} \\
n=n_{1}+n_{2}+\cdots n_{l}
\end{array}\right.
$$

with positive integers $m_{i}$ and $n_{j}, 1 \leqq i \leqq k, 1 \leqq j \leqq l$, where $k$ and $l$ are subject to $1 \leqq k$ $\leqq m, 1 \leqq l \leqq n$.

$\left(2^{\circ}\right)$ In correspondence with the pair of partitions given in $\left(1^{\circ}\right)$, the whole $m \times n$ cell space is divided into kl subspaces, each of which will be denotea by $S\left(m_{i}, n_{j}\right)(i=$ $1,2, \cdots, k ; j=1,2, \cdots, l)$.

$\left(3^{\circ}\right)$ (a) The elements of each subspace $S\left(m_{i}, n_{j}\right)$ are entirely either 1 or zero.

A subspace $S\left(m_{i}, n_{j}\right)$ all of whose elements are equal to 1 is called to be of Type 1, while a subspace all of whose elements are equal to zero to be of Type 0.

(b) Subspaces of these two types occur alternatively in an $m \times n$ cell space.

Furthermore we observe

COROLlary 2.2. We have

where

$$
\begin{aligned}
& C_{b}^{a}(m, n)=\left(\begin{array}{cccccc}
a_{1} & b_{2} & \cdots & b_{j} & \cdots & b_{n} \\
a_{2} & a_{2}+b_{2} & \cdots & a_{2}+b_{j} & \cdots & a_{2}+b_{n} \\
\cdots \cdots \cdots \cdots \cdots \cdots \cdots \cdots \cdots \cdots \cdots \cdots \cdots & \cdots \cdots \cdots \cdots \\
a_{i} & a_{i}+b_{2} & \cdots & a_{i}+b_{j} & \cdots & a_{i}+b_{n} \\
\cdots \cdots \cdots \cdots \cdots \cdots \cdots \cdots \cdots \cdots \cdots \cdots \cdots & \cdots \cdots \cdots \cdots \\
a_{m} & a_{m}+b_{2} & \cdots & a_{m}+b_{j} & \cdots & a_{m}+b_{n}
\end{array}\right) \\
& +\left(\begin{array}{cccccc}
0 & 0 & \cdots & 0 & \cdots & 0 \\
0 & z_{22} & \cdots & z_{2 j} & \cdots & z_{2 n} \\
\cdots \cdots \cdots \cdots \cdots & \cdots \cdots \cdots \\
0 & z_{i 2} & \cdots & z_{i j} & \cdots & z_{i n} \\
\cdots & \cdots \cdots \cdots \cdots \cdots \cdots \\
0 & z_{m 2} & \cdots & z_{m j} & \cdots & z_{m n}
\end{array}\right)
\end{aligned}
$$

$$
z_{i j} \equiv \sum_{p=2}^{t} \sum_{q=2}^{j} x_{p q} \quad(\bmod .2)
$$

This COROLlary 2.2 implies that $C_{b}^{a}(m, n)$ can be expressed as the sum of two matrices one of which is the stable configuration which is uniquely determined by the boundary condition $B d(a, b)$, while the other one is $\triangle_{0}^{0} X$ induced by $X$ in (2.01) with the boundary condition $B(0,0)$.

It is to be noted that

COROLlARY 2.3. The correspondence between two matrices $X=\left(x_{i j}\right)(i=2,3, \cdots$, $m ; j=2,3, \cdots, n)$ and $Z=\left(z_{i j}\right)(i=2,3, \cdots, m ; j=2,3, \cdots, n)$ is one-to-one.

Because of COROLlaries 2.2 and 2.3 , all the possible configurations $C_{b}^{a}(m, n)$ can be obtained by running through all the possible boundary conditions $B d(a, b)$ and all the possible $(m-1) \times(n-1)$ matrices with elements 1 or 0 . 


\section{Proof of the Theorem I}

In view of COROLlary 2.2, let us denote the first and the second matrices in the right-hand side in (2.08) by $S_{b}^{a}$ and $P_{z}$ respectively. We may and we shall sometimes write $P_{x}^{*}$ instead of $P_{z}$ because of the one-to-one correspondence between $x$ and $z$ as shown in COROLLARY 2.3 .

Now (2.08) amounts to the equality

$$
C_{b}^{a}(m, n)=S_{b}^{a}+P_{z}=S_{b}^{a}+P_{x}^{*}
$$

Let us consider the set of all the possible sets of $\left(a_{1}, a_{2}, \cdots, a_{m} ; b_{2}, b_{3}, \cdots, b_{n}\right)$ :

$$
\begin{aligned}
B d(m, n-1)= & {\left[\left(a_{1}, a_{2}, \cdots, a_{m} ; b_{2}, b_{3}, \cdots, b_{n}\right) ;\right.} \\
& \left.a_{i}, b_{j}=1,0,(i=1 \sim m ; j=2 \sim n)\right] .
\end{aligned}
$$

Furthermore let us consider the set of all the possible configurations

$$
K_{1}(m-1, n-1)=\left[\left(z_{i j}\right) ; z_{i, j}=1,0,(i=2 \sim m ; j=2 \sim n)\right] .
$$

For the sake of systematic enumerations of all elements in $B d(m, n-1)$ and of those in $K_{1}(m-1, n-1)$, let us introduce

$$
\begin{aligned}
u_{i} & =a_{i} \quad(i=1,2, \cdots, m) \\
& =b_{(i-m)+1} \quad(i=m+1, m+2, \cdots, m+n-1),
\end{aligned}
$$

and

$$
v_{j}=z_{p, q}, \quad \text { for } j=(m-1)(p-2)+q-1,
$$

where $p=2 \sim m, q=2 \sim n$.

Then we may and we shall write

$$
\begin{gathered}
B d(m, n-1)=\left[\left(u_{1}, u_{2}, \cdots, u_{m \div n-1}\right) ; u_{i}=1,0(i=1,2, \cdots, m+n-1)\right] \\
K_{1}(m-1, n-1)=\left[\left(v_{1}, v_{2}, \cdots, v_{(m-1)(n-1)} ; v_{i}=1,0\right.\right. \\
(i=1,2, \cdots,(m-1)(n-1))] .
\end{gathered}
$$

Now the lexicographical representations of all elements in (3.06) can be given as follows:

\begin{tabular}{cccccccc}
$B d(m, n-1)$ & & \multicolumn{1}{l}{} \\
& $u_{1}$ & $u_{2}$ & $u_{3}$ & $\cdots$ & $u_{m+n-3}$ & $u_{m \div \cdot n-2}$ & $u_{m+-n-1}$ \\
$(1)$ & 0 & 0 & 0 & $\cdots$ & 0 & 0 & 0 \\
$(2)$ & 0 & 0 & 0 & $\cdots$ & 0 & 0 & 1 \\
$(3)$ & 0 & 0 & 0 & $\cdots$ & 0 & 1 & 0 \\
$(4)$ & 0 & 0 & 0 & $\cdots$ & 0 & 1 & 1 \\
$(5)$ & 0 & 0 & 0 & $\cdots$ & 1 & 0 & 0 \\
$(6)$ & 0 & 0 & 0 & $\cdots$ & 1 & 0 & 1 \\
$(7)$ & 0 & 0 & 0 & $\cdots$ & 1 & 1 & 0 \\
$(8)$ & 0 & 0 & 0 & $\cdots$ & 1 & 1 & 1 \\
$\vdots$ & $\vdots$ & $\vdots$ & $\vdots$ & & $\vdots$ & $\vdots$ & $\vdots$ \\
$\left(2^{m+n-2}\right)$ & 0 & 1 & 1 & $\cdots$ & 1 & 1 & 1
\end{tabular}


Let us denote by $u^{(i)}=\left(u_{2}^{(i)}, u_{3}^{(i)}, \cdots, u_{m+n-1}^{(i)}\right)$ the element of $B d(m, n-1)$ whose number of listing is $i$ in the above lexicographical listing. It is noted that $\bar{u}^{(i)} \equiv$ $\left(\bar{u}_{2}^{(i)}, \bar{u}_{3}^{(i)}, \cdots, \bar{u}_{m+n-1}^{(i)}\right)$, that is the conjugate of $u^{(i)}$, is equal to $u^{g(i)}$ with $g(i)=2^{(m+n-2)}$ $-(i-1)$ for $i=1,2, \cdots, m+n-2$.

Similarly the inverse lexicographical representation of all elements in (3.07) can be given as follows:

\begin{tabular}{|c|c|c|c|c|c|c|c|}
\hline \multicolumn{8}{|c|}{$K_{1}(m-1, n-1)$} \\
\hline & $v_{1}$ & $v_{2}$ & $v_{3}$ & $\cdots$ & $v_{(m-1)(n-1)-2}$ & $v_{(m-1)(n-1)-1}$ & $v_{(m-1)(n-1)}$ \\
\hline (1) & 1 & 1 & 1 & $\cdots$ & 1 & 1 & 1 \\
\hline (2) & 1 & 1 & 1 & $\ldots$ & 1 & 1 & 0 \\
\hline (3) & 1 & 1 & 1 & $\cdots$ & 1 & 0 & 1 \\
\hline (4) & 1 & 1 & 1 & $\ldots$ & 1 & 0 & 0 \\
\hline (5) & 1 & 1 & 1 & $\cdots$ & 0 & 1 & 1 \\
\hline (6) & 1 & 1 & 1 & $\ldots$ & 0 & 1 & 0 \\
\hline$(7)$ & 1 & 1 & 1 & $\cdots$ & 0 & 0 & 1 \\
\hline$\left(2^{(m-1)(n-1)-1}\right)$ & 1 & 0 & 0 & $\ldots$ & 0 & 0 & 0 \\
\hline$\left(2^{(m-1)(n-1)}+1\right)$ & 0 & 1 & 1 & $\cdots$ & 1 & 1 & 1 \\
\hline
\end{tabular}

By means of these lexicographical listings, let us denote

$$
S_{b}^{a}=S^{(j)} \quad\left(j=1,2, \cdots, 2^{m \cdot{ }^{n-1}}\right)
$$

for each element $(a, b)$ in $B d(m, n-1)$ which corresponds to $u^{(j)}$, and

$$
P_{z}=P^{(i)} \quad\left(i=1,2, \cdots, 2^{(m, n)}\right)
$$

for each element $z$ in $K_{1}(m-1, n-1)$ which corresponds to $v^{(i)}$. Now let us introduce

$$
K^{(i)}(m, n) \equiv\left[S^{(j)}+P^{(i)} ; j=1,2, \cdots, m+n-1\right]
$$

for $i=1,2, \cdots,(m-1)(n-1)$.

After these preparations let us turn to the proof of our main THEorem I enunciated in Section 1.

Now it is evident that

$$
\sum_{i=1}^{\varphi(m, n)} K^{(i)}(m, n)=K(m, n)
$$

and also that

$$
K^{(i)}(m, n) K^{(j)}(m, n)=\boldsymbol{\phi}
$$

for $i \neq j$, because any elemert in $K(m, n)$ must have one and only one combination of $\left(u_{i}, v_{j}\right)$ and for each combination $(i, j)$ there exists one and only one element in $K(m, n)$, as was to prove.

Furthermore it is noted that $K^{(\varphi(m, n))}(m, n)$ is the set of all the stable configuration, because $P^{(\varphi(m, n))}$ is the $m \times n$ configuration matrix whose elements are all zero. On the other hand $K^{(1)}(m, n)$ is the set of all the configurations in an $m \times n$ cell space 
which belong to the Eden generation defined in Definition 1.1, because $P^{(1)}$ has, for example, the form

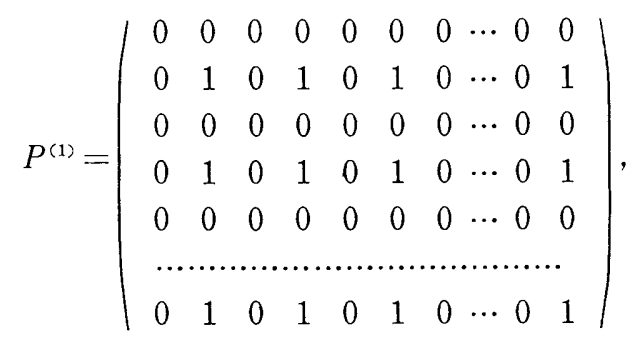

which amounts to say that every $(m-1)(n-1)$ possible firing point yields a change of each basic cell associated with it and that the two conditions given in DEFINITION 1.1 are satisfied.

\section{Examples}

EXAMPle 4.1. $2 \times 2$ cell space. Since $m=2, n=2$, the fundamental numbers $m+n-1=3$ and $(m-1)(n-1)=1$ give that there are $2^{3}=8$ stable configurations and that the set of all the configurations $K(2,2)$ is the sum of the two subsets $K^{(1)}(2,2)$ and $K^{(2)}(2,2)$ where the former is the set of all 8 stable configurations and the latter the set of all 8 configuration belonging to the Eden generation. An application of of general considerations given in Sections 2 and 3 yield us the following details:

$\left(1^{\circ}\right)$ The lexicographical listing of $u=\left(u_{1}, u_{2}, u_{3}, u_{4}\right)$

$\begin{array}{lccccccc} & u_{1} & u_{2} & u_{3} & & u_{1} & u_{2} & u_{3} \\ \text { (1) } & 0 & 0 & 0 & (5) & 1 & 0 & 0 \\ (2) & 0 & 0 & 1 & (6) & 1 & 0 & 1 \\ \text { (3) } & 0 & 1 & 0 & (7) & 1 & 1 & 0 \\ \text { (4) } & 0 & 1 & 1 & (8) & 1 & 1 & 1\end{array}$

$\left(2^{\circ}\right)$ The set of all 8 stable configurations $K(2,2)$. In correspondence with $\left(1^{\circ}\right)$, we have
(1) $\left(\begin{array}{ll}0 & 0 \\ 0 & 0\end{array}\right)=C_{1}$
(2) $\left(\begin{array}{ll}0 & 1 \\ 0 & 1\end{array}\right)=C_{2}$
(3) $\left(\begin{array}{ll}0 & 0 \\ 1 & 1\end{array}\right)=C_{3}$
(4) $\left(\begin{array}{ll}0 & 1 \\ 1 & 0\end{array}\right)=C_{4}$
(5) $\left(\begin{array}{ll}1 & 0 \\ 0 & 1\end{array}\right)=C_{5}$
(6) $\left(\begin{array}{ll}1 & 1 \\ 0 & 0\end{array}\right)=C_{6}$
(7) $\left(\begin{array}{ll}1 & 0 \\ 1 & 0\end{array}\right)=C_{7}$
(8) $\left(\begin{array}{ll}1 & 1 \\ 1 & 1\end{array}\right)=C_{8}$

$\left(3^{\circ}\right)$ The set of all 8 configurations belonging to the Eden generation $K^{(1)}(2,2)$. The Boolean matrix addition of the matrix

$$
P_{2}^{*}=\left(\begin{array}{ll}
0 & 0 \\
0 & 1
\end{array}\right)
$$

to each of the matrix (4.01) gives us the set of all 8 configurations belonging ${ }^{-}$to the Eden generation 
(4.03)
(1) $\left(\begin{array}{ll}0 & 0 \\ 0 & 1\end{array}\right)=C_{1}^{(1)}$
(2) $\left(\begin{array}{ll}0 & 1 \\ 0 & 0\end{array}\right)=C_{2}^{(1)}$
(3) $\left(\begin{array}{ll}0 & 0 \\ 1 & 0\end{array}\right)=C_{3}^{(1)}$
(4) $\left(\begin{array}{ll}0 & 1 \\ 1 & 1\end{array}\right)=C_{4}^{(1)}$
(5) $\left(\begin{array}{ll}1 & 0 \\ 0 & 0\end{array}\right)=C_{5}^{(1)}$
(6) $\left(\begin{array}{ll}1 & 1 \\ 0 & 1\end{array}\right)=C_{6}^{(1)}$
(7) $\left(\begin{array}{ll}1 & 0 \\ 1 & 1\end{array}\right)=C_{7}^{(1)}$
(8) $\left(\begin{array}{ll}1 & 1 \\ 1 & 0\end{array}\right)=C_{8}^{(1)}$.

$\left(4^{\circ}\right)$ The transition aspects in $K(2,2)$ There are merely two subsets $K^{(1)}(2,2)$ (Eden) and $K^{(2)}(2,2)$ (stable).

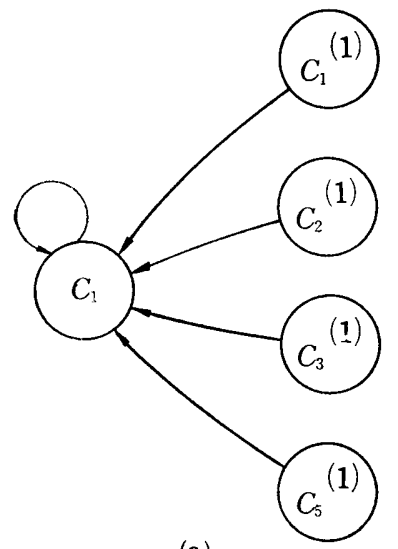

(a)

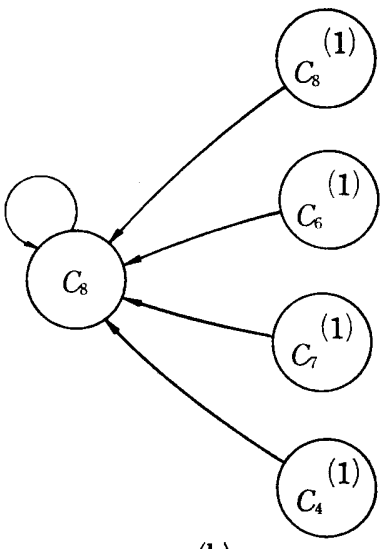

(b)

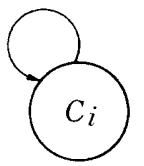

( $\mathrm{i}=2,3,4$, $5,6,7)$.

(c)

Fig. 4.1, (a), (b), (c). Genealogical lineages in the $2 \times 2$ cell space.

It is to be noted that the mutual conjugate relations hold true

$$
\begin{array}{ll}
\bar{C}_{i}=C_{9-i} & (i=1,2, \cdots, 8) \\
\bar{C}_{i}^{(1)}=\bar{C}_{9-i}^{(1)} & (i=1,2, \cdots, 8) .
\end{array}
$$

Thus Figure 4.1, (b) is a conjugate picture of Figure 4.1, (a). Figure 4.1, (c) consists of two mutually conjugate pictures of isolated stable corfigurations.

EXAMPle 4.2. $2 \times 3$ cell space. This is the case when $m=2$ and $n=8$. The procedure similar to EXAMPLE 2.1 gives us

$\left(1^{\circ}\right)$ The lexicographical listing of $u=\left(u_{1}, u_{2}, u_{3}, u_{4}\right)$. This consists the set of $2^{4}$ vectors which can be divided into two mutually conjugate subset each consisting of $2^{2}$ vectors.

$\left(2^{\circ}\right)$ The set of all 16 stable configurations $K^{(4)}(2,3)$. This consists of two subsets. The first subset consists of
(1) $\left(\begin{array}{lll}0 & 0 & 0 \\ 0 & 0 & 0\end{array}\right)=C_{1}^{(4)}$
(2) $\left(\begin{array}{lll}0 & 0 & 1 \\ 0 & 0 & 1\end{array}\right)=C_{2}^{(4)}$
(3) $\left(\begin{array}{lll}0 & 1 & 0 \\ 0 & 1 & 0\end{array}\right)=C_{3}^{(4)}$
(4) $\left(\begin{array}{lll}0 & 1 & 1 \\ 0 & 1 & 1\end{array}\right)=C_{4}^{(4)}$
(5) $\left(\begin{array}{lll}0 & 0 & 0 \\ 1 & 1 & 1\end{array}\right)=C_{5}^{(4)}$
(6) $\left(\begin{array}{lll}0 & 0 & 1 \\ 1 & 1 & 0\end{array}\right)=C_{6}^{(4)}$ 


$$
\text { (7) }\left(\begin{array}{lll}
0 & 1 & 0 \\
1 & 0 & 1
\end{array}\right)=C_{7}^{(4)} \quad \text { (8) }\left(\begin{array}{lll}
0 & 1 & 1 \\
1 & 0 & 0
\end{array}\right)=C_{8}^{(4)} \text {, }
$$

while the second subset consists of the conjugates of the stable configurations belonging to the first subset, namely $\bar{C}_{i}^{(4)}(i=1,2,3,4,5,6,7,8)$.

$\left(3^{\circ}\right)$ The lexicographical listing of $v=\left(v_{1}, v_{2}\right)$ gives us

$\begin{array}{lccccc} & v_{1} & v_{2} & & v_{1} & v_{2} \\ \text { (1) } & 1 & 1 & \text { (3) } & 0 & 1 \\ \text { (2) } & 1 & 0 & \text { (4) } & 0 & 0 .\end{array}$

Consequently we have

$$
\begin{aligned}
& K^{(1)}(2,3)=\left[S^{u}+\left(\begin{array}{lll}
0 & 0 & 0 \\
0 & 1 & 1
\end{array}\right) ; u \in B d(2,2)\right] \\
& K^{(2)}(2,3)=\left[S^{u}+\left(\begin{array}{lll}
0 & 0 & 0 \\
0 & 1 & 0
\end{array}\right) ; u \in B d(2,2)\right] \\
& K^{(3)}(2,3)=\left[S^{u}+\left(\begin{array}{lll}
0 & 0 & 0 \\
0 & 0 & 1
\end{array}\right) ; u \in B d(2,2)\right] \\
& K^{(4)}(2,3)=\left[S^{u}+\left(\begin{array}{lll}
0 & 0 & 0 \\
0 & 0 & 0
\end{array}\right) ; u \in B d(2,2)\right] .
\end{aligned}
$$

The systematic enumerations give us

$$
K^{(i)}(2,3)=\left[C_{j}^{(\imath)}, \vec{C}_{j}^{(i)} ; j=1,2,3, \cdots, 8\right],
$$

where

$$
C_{j}^{(i)}=C_{j}^{(4)}+P * v^{(i)}
$$

with

$$
\begin{cases}v^{(1)}=(1,1) & v^{(2)}=(1,0) \\ v^{(3)}=(0,1) & v^{(4)}=(0,0) .\end{cases}
$$

$\left(4^{\circ}\right)$ Each of the following sixteen stable configurations is an isolated stable configuration.

$$
\begin{array}{lllll}
C_{3}^{(4)}, & C_{5}^{(4)}, & C_{6}^{(4)}, & C_{7}^{(4)}, & C_{8}^{(4)} \\
\overline{C_{3}^{(4)}}, & \overline{C_{5}^{(4)}}, & \overline{C_{6}^{(4)}}, & \overline{C_{7}^{(4)}}, & \overline{C_{8}^{(4)}} .
\end{array}
$$

$\left(5^{\circ}\right)$ For each non-isolated stable configuration we show its genealogy until we find out all its ancestors.

(a) $C_{1}^{(4)}=\left(\begin{array}{lll}0 & 0 & 0 \\ 0 & 0 & 0\end{array}\right)$. This has the following six direct ancestors, among which two have no ancestors, and are in fact belonging to the Eden generation, as shown by $(E)$.
(1.1) $\left(\begin{array}{lll}0 & 0 & 1 \\ 0 & 0 & 0\end{array}\right)$
(1.2) $\left(\begin{array}{lll}0 & 0 & 0 \\ 0 & 0 & 1\end{array}\right)$
(1.3) $\left(\begin{array}{lll}0 & 0 & 0 \\ 0 & 1 & 0\end{array}\right)$
(E) (1.4) $\left(\begin{array}{lll}0 & 0 & 0 \\ 1 & 0 & 0\end{array}\right)$
(1.5) $\left(\begin{array}{lll}1 & 0 & 0 \\ 0 & 0 & 0\end{array}\right)$
(1.6) $\left(\begin{array}{lll}0 & 1 & 0 \\ 0 & 0 & 0\end{array}\right)$
(E). 
We proceed to find out the set of all the direct ancestors for (1.1) in (4.15).

$$
\begin{aligned}
& \text { (1.1.1) }\left(\begin{array}{lll}
0 & 1 & 1 \\
0 & 0 & 0
\end{array}\right)(N A) \quad(1.1 .2)\left(\begin{array}{lll}
0 & 0 & 1 \\
0 & 1 & 0
\end{array}\right)(N A) \\
& \text { (1.1.3) }\left(\begin{array}{lll}
0 & 0 & 1 \\
1 & 0 & 0
\end{array}\right)(E) \quad(1.1 .4)\left(\begin{array}{lll}
1 & 0 & 1 \\
0 & 0 & 0
\end{array}\right)(E) \text {, }
\end{aligned}
$$

where configurations having no direct ancestor are denoted by $(N A)$.

There are simple transformations $S_{i}(i=1,2)$ such that

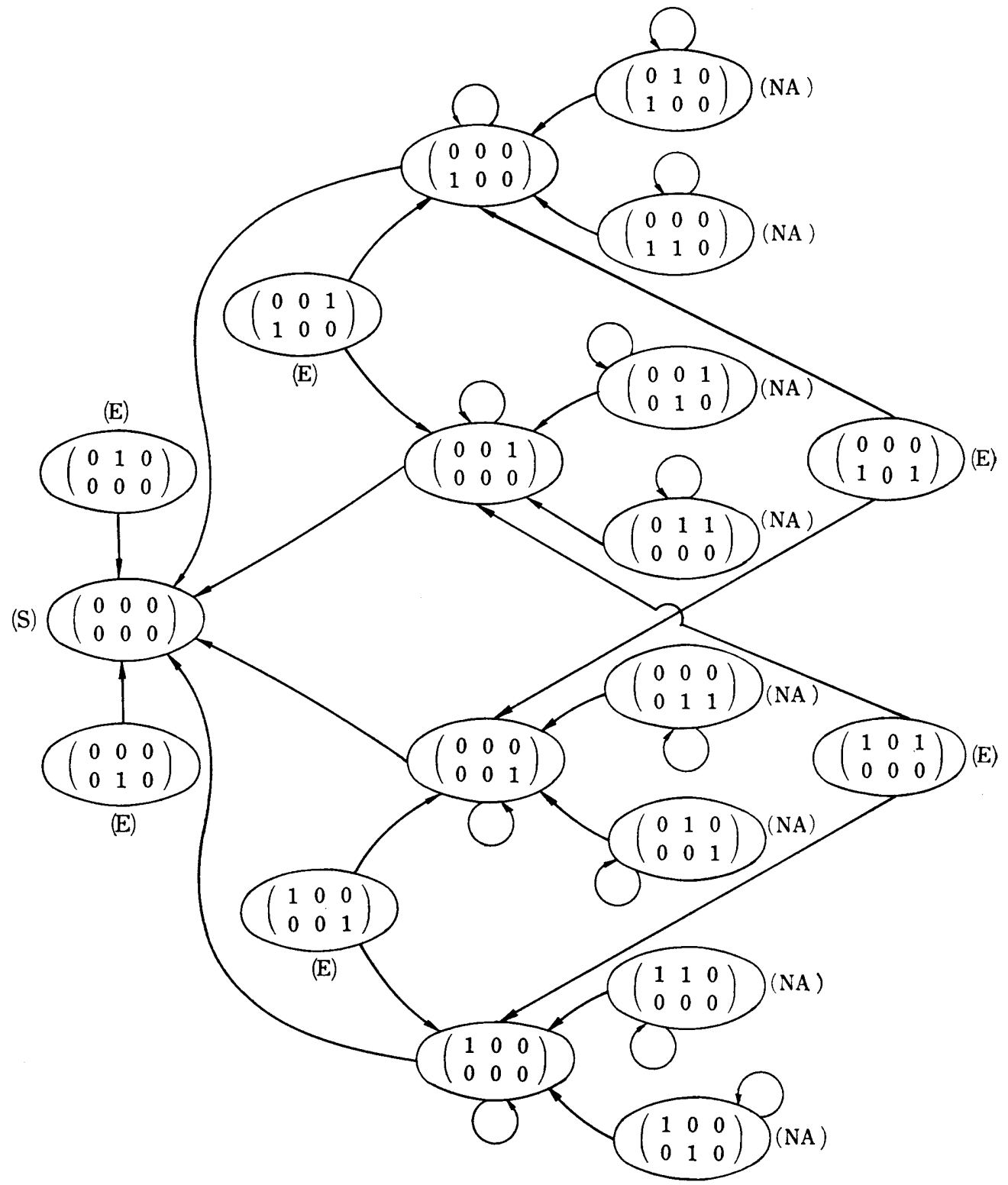

Fig. 4.2, (a). Genealogical lineage in the $2 \times 3$ cell space (1). 


$$
S_{1}\left(\begin{array}{lll}
0 & 0 & 1 \\
0 & 0 & 0
\end{array}\right)=\left(\begin{array}{ccc}
0 & 0 & 0 \\
0 & 0 & 1
\end{array}\right), \quad S_{2}\left(\begin{array}{lll}
0 & 0 & 1 \\
0 & 0 & 0
\end{array}\right)=\left(\begin{array}{ccc}
0 & 0 & 0 \\
1 & 0 & 0
\end{array}\right),
$$

which give us immediately direct ancestors for (1.2), and (1.5), in view of (5.16).

(b) $C_{2}^{(4)}=\left(\begin{array}{lll}0 & 0 & 1 \\ 0 & 0 & 1\end{array}\right)$. This has the following four direct ancestors.
(2.1) $\left(\begin{array}{lll}0 & 1 & 1 \\ 0 & 0 & 1\end{array}\right)(E)$
(2.2) $\left(\begin{array}{lll}0 & 0 & 1 \\ 0 & 1 & 1\end{array}\right)(E)$
(2.3) $\left(\begin{array}{lll}0 & 0 & 1 \\ 1 & 0 & 1\end{array}\right)(N A)$
(2.4) $\left(\begin{array}{lll}1 & 0 & 1 \\ 0 & 0 & 1\end{array}\right)(N A)$

Each of these four configurations has no direct ancestor.

(c) $C_{4}^{(4)}=\left(\begin{array}{lll}0 & 1 & 1 \\ 0 & 1 & 1\end{array}\right)$. This has the following four direct ancestors.
(4.1) $\left(\begin{array}{lll}0 & 1 & 0 \\ 0 & 1 & 1\end{array}\right)(E)$
(4.2) $\left(\begin{array}{lll}0 & 1 & 1 \\ 0 & 1 & 0\end{array}\right)(N A)$
(4.3) $\left(\begin{array}{lll}0 & 1 & 1 \\ 0 & 0 & 1\end{array}\right)(E)$
(4.4) $\left(\begin{array}{ccc}0 & 0 & 1 \\ 1 & 1 & 1\end{array}\right)(N A)$.

Again here each of these four configurations has no direct ancestor.

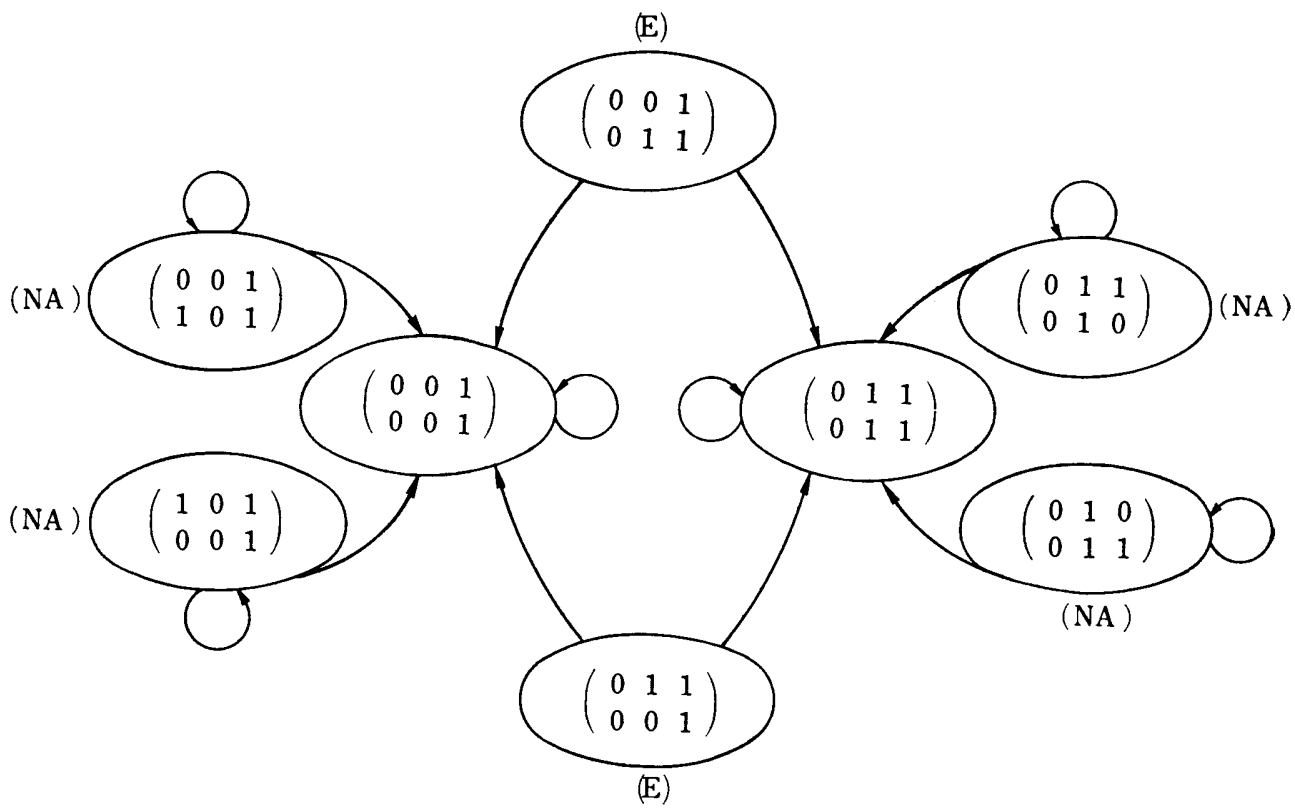

Fig. 4.2, (b). Genealogical lineage in the $2 \times 3$ cell space (2).

\section{Genealogical classification of all the configurations in an $m \times n$ cell space}

In an $m \times n$ cell space there exists a set of $(m-1)(n-1)$ firing points $\left\{q_{i, j}\right\} \quad(i=1$, $2, \cdots, m-1 ; j=1,2, \cdots, n-1)$. Let us denote the local majority transformation applied at the firing points $q_{i, j}$ by $T(i, j)$. To each pair of assigned configuration $C$ 
and LMT $T(i, j)$ in an $m \times n$ cell space we are concerned with the result of application of $T(i, j)$ to the configuration $C$, that is, $T(i, j) C$ and also with the solution of finding another configuration $C^{\prime}$ such that $T(i, j) C^{\prime}=C$ for the assigned configuration C. The location of each $q_{i, j}$ can be illustrated in the following Figure 5.1.

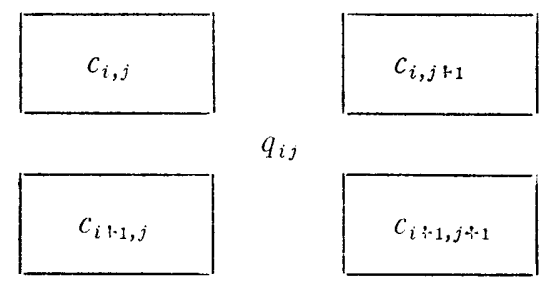

Fig. 5.1. The location of the firing point $q_{i, j}$.

Now the following two observations are sufficient enough to obtain all the direct descendants and ancestors of any given configuration $C$.

ObSERVATION (a): At each firing point $q_{i j}$ for which

$$
\left.y_{i, j}+y_{i: 1, j}+y_{i, j+1}+y_{i+1, j: 1} \equiv 1 \quad \text { (mod. } 2\right),
$$

$T(i, j) C$ gives a direct descendant of $C$ which is different from the configuration $C$, but $C$ has no direct ancestor for which $T(i, j) C^{\prime}=C$.

OBSERVATION (b): At each firing point $q_{i j}$ for which

$$
y_{i, j}+y_{i+1, j}+y_{i, j \div 1}+y_{i+1, j \div 1} \equiv 0 \quad(\bmod .2),
$$

$T(i, j) C$ is coincident with $C$. Regarding direct ancestors of $C$, there are two distinct cases (i) and (ii):

(i) When just two cells among the four cells have the value 1 and the other two cells have the value 0 , then there is no direct ancestor $C^{\prime}$ such that $T(i, j) C^{\prime}=C$.

(ii) When each of all the four cells has the same value, either of 1 and 0 , then there are four direct ancestors $C^{\prime}$ such that $T(i, j) C^{\prime}=C$.

We now introduce

Definition 5.1. A stable configuration is said to be isolated under the LMT if it has no direct ancestor.

The set of all the isolated stable configurations in an $m \times n$ cell space is denoted by $S_{0}(m, n)$, and hence the set of all the non-isolated stable configurations in an $m \times n$ cell space is $K_{\varphi(m, n)}(m, n)-S_{0}(m, n)$.

DeFinition 5.2. The sequence $\left\{d^{(\nu)}(A)\right\}(\nu=1,2,3, \cdots)$ of configurations defined by

$$
d^{(\nu)}(A)=d\left(d^{(\nu-1)}(A)\right), \quad(\nu=2,3, \cdots)
$$

with the relations

$$
d^{(1)}(A)=d(A), \quad d^{(0)}(A)=A .
$$

is called to be the sequence of descendants of a configuration $A$ in an $m \times n$ cell space.

DEFINITION 5.3. The sequence $\left\{a^{(\nu)}(A)\right\}(\nu=1,2,3, \cdots)$ of configurations defined by 


$$
a^{(2)}(A)=a\left(a^{(\nu-1)}(A)\right) \quad(\nu=2,3, \cdots)
$$

with the relations

$$
a^{(1)}(A)=a(A), \quad a^{(0)}(A)=A
$$

is called to be the sequence of ancestors of a configuration $A$ in an $m \times n$ cell space.

In view of these Definitions we have

COROLLARY 5.1. For any configuration $A$ there is a pair of non-negative integers $N_{d}(A)$ and $N_{a}(A)$ depending up the configuration $A$ such that

$$
\begin{aligned}
& \mathfrak{D}(A)=\sum_{\nu=0}^{N_{d(A)}} d^{(\nu)}(A) \\
& \mathfrak{Y}(A)=\sum_{\nu=0}^{N_{d^{(}(A)}} a^{(\nu)}(A) .
\end{aligned}
$$

Now a systematic procedure for determining a genealogical classification of all the configurations in an $m \times n$ cell space can be given in the following way.

$\left(1^{\circ}\right)$ By means of THEOREM 3 in [1], the set of all the possible $2^{m+n-1}$ stable configurations $K_{\varphi(m, n)}(m, n)$ in an $m \times n$ cell space can be given in a systematic way, by a lexicographical listing of all the possible combinations of $\left\{m_{i}\right\}(i=1,2,3, \cdots)$ and $\left\{n_{j}\right\}(j=1,2, \cdots)$ given in (2.07).

$\left(2^{\circ}\right)$ In view of THEOREM 1 in [2], all the possible isolated stable configurations can be enumerated systematically. In fact a stable configuration is isolated if and only if at least one of the following two conditions (i) and (ii) is satisfied for (2.07):

(i) $\quad m_{1}=m_{2}=\cdots=m_{k}=1$

(ii) $n_{1}=n_{2}=\cdots=n_{l}=1$.

$\left(3^{\circ}\right)$ In view of the observations given above, we can determine $a^{(\nu)}(S)(\nu=1$, $\left.2,3, \cdots, N_{a}(A)\right)$ and hence

$$
\mathfrak{R}(S)=\sum_{\nu=0}^{N_{\alpha}(A)} a^{(\nu)}(S)
$$

to each assigned $S$ belonging to $K_{\varphi}(m, n)-S_{0}(m, n)$.

$\left(4^{\circ}\right)$ In view of the observations given above, we can determine $d^{(\nu)}(B)(\nu=1$, $\left.2,3, \cdots, N_{d}(B)\right)$ and hence

$$
\mathfrak{D}(B)=\sum_{\nu=0}^{N d^{(B)}} d^{(\nu)}(B)
$$

to each assigned $B$ belonging to $\mathfrak{\Re}(S)$.

$\left(5^{\circ}\right)$ We construct

$$
\mathfrak{L}(S)=[\mathfrak{D}(B) ; B \in \mathfrak{Y}(S)] .
$$

$\left(6^{\circ}\right)$ Let us choose any $S_{1}$ belonging to $K_{\varphi(m, n)}(m, n)-S_{0}(m, n)$.

$$
\begin{gathered}
S\left(S_{1}\right)=K_{\varphi(m, n)}(m, n) \cap \mathbb{2}\left(S_{1}\right) \\
R K_{\varphi}\left(S_{1}\right)=K_{\varphi(m, n)}(m, n)-S_{0}(m, n)-S\left(S_{1}\right) .
\end{gathered}
$$

Let us choose a configuration $S_{2}$ belonging to $R K_{\varphi}\left(S_{1}\right)$. 
$\left(7^{\circ}\right)$ Proceeding on $S_{2}$ similarly, as we have done for $S_{1}$ in $\left(3^{\circ}\right) \sim\left(5^{\circ}\right)$, we construct $\mathbb{L}(B)$, and hence we define

$$
\begin{gathered}
S\left(S_{2}\right)=K_{\varphi(m, n)}(m, n) \cap \mathfrak{R}\left(S_{2}\right) \\
R K_{\varphi}\left(S_{1}, S_{2}\right)=R K_{\varphi}\left(S_{1}\right)-S\left(S_{2}\right) .
\end{gathered}
$$

Then let us choose a configuration $S_{3}$ belonging to $R K_{\varphi}\left(S_{1}, S_{2}\right)$.

$\left(8^{\circ}\right)$ The process indicated in $\left(7^{\circ}\right)$ will be repeated until a sequence of nonisolated stable configurations $\left\{S_{i} ; i=1,2, \cdots, k\right\}$ can be found which satisfies the following conditions.

$$
\begin{aligned}
& \text { (i) } \mathfrak{L}\left(S_{i}\right) \cap \mathfrak{L}\left(S_{j}\right)=\phi \quad(i \neq j ; i, j=1,2, \cdots, k) \\
& \text { (ii) } \mathfrak{L}\left(S_{i}\right) \cap S_{0}(m, n)=\phi \quad(i=1,2, \cdots, k) \\
& \text { (iii) } R K_{\varphi(m, n)}\left(A_{1}, A_{2}, \cdots, A_{\nu}\right) \neq \phi \quad(\nu=1,2, \cdots, k-1) \\
& \text { (iv) } R K_{\varphi(m, n)}\left(A_{1}, A_{2}, \cdots, A_{k}\right)=\phi \\
& \text { (v) } K_{\varphi(m, n)}(m, n)=S_{0}(m, n)+\sum_{i=1}^{k} S\left(S_{i}\right) \\
& \text { (vi) } K(m, n)=S_{0}(m, n)+\sum_{i=1}^{k} \mathfrak{L}\left(S_{i}\right) .
\end{aligned}
$$

The construction procedure just given yields us the following result, which we may and we shall call a genealogical classification Lemma regarding the set of all the possible configurations in an $m \times n$ cell space.

LEMMA 1. In the set of all the $2^{m n}$ possible configurations in an $m \times n$ cell space under LMT we can find a set of finite numbers of stable configurations $\left\{S_{i} ; i=1,2\right.$, $\cdots, g\}$ such that

$\left(1^{\circ}\right) \quad \mathfrak{L}\left(S_{i}\right) \cap \mathfrak{L}\left(S_{j}\right)=\phi($ for $i \neq j ; i, j=1,2, \cdots, g)$

$\left(2^{\circ}\right)$ For a certain positive integer $h(<g)$, we have

$$
\mathfrak{L}\left(S_{i}\right)=\left\{S_{i}\right\} \quad(i=1,2,3, \cdots, h)
$$

$\left(3^{\circ}\right) \quad K(m, n)=\sum_{i=1}^{g} \mathfrak{L}\left(S_{i}\right)$

$\left(4^{\circ}\right) \quad K_{\varphi(m, n)}(m, n)=\sum_{i=1}^{g} K\left(S_{i}\right)$

where

$$
K\left(S_{i}\right)=\mathfrak{L}\left(S_{i}\right) \cap K_{\varphi(m, n)}(m, n) \quad(i=1,2, \cdots, g) .
$$

In performing the procedure given above, there are several considerations which are required to overcome the complex multiplitude caused by the existence of tremendous number of all the possible configurations in an $m \times n$ cell space amounting to be $2^{m n}$. At least two considerations are necessary for treating with even simple cases such as $3 \times 3$ and $4 \times 4$ and so on. The first one is to take into our consideration that rotation group inherited in the sets of configuration in an $n \times n$ cell space and induced by a rotation $\theta$ such that 


$$
\left(\begin{array}{cccc}
a_{11} & a_{12} & \cdots & a_{1 n} \\
a_{21} & a_{22} & \cdots & a_{2 n} \\
a_{31} & a_{32} & \cdots & a_{3 n} \\
\vdots & \vdots & & \vdots \\
a_{n 1} & a_{n 2} & \cdots & a_{n n}
\end{array}\right)=\left(\begin{array}{cccc}
a_{n 1} & a_{n-1} & \cdots & a_{11} \\
a_{n 2} & a_{n-1} & \cdots & a_{12} \\
a_{n 3} & a_{n-1} & \cdots & a_{13} \\
\vdots & \vdots & & \vdots \\
a_{n n} & a_{n-1} & \cdots & a_{1 n}
\end{array}\right) .
$$

In fact ${ }_{2}^{*}$ there are three groups induced by $\theta$ :

$$
\begin{aligned}
& \Theta=\left\{1, \theta, \theta^{2}, \theta^{3}\right\} \\
& \Phi=\left\{1, \theta^{2}\right\} \\
& \Psi=\{1\}
\end{aligned}
$$

where $\theta^{4}=1$ (identitical transformation).

The rotation group considerations make our enumeration works and findings of transition relations among configurations simpler by making no distinction among the configurations belonging to the same $\theta$-group such as $\Theta$ and $\Phi$.

This implies that we are actually concerned with structural aspect of configurations from the standpoint of category rather than with each individual configurations.

The second one is to be pragmatic in choosing a set of $\left\{S_{i}\right\}$ in LEMma 1 in the sense that $\mathfrak{I}\left(S_{i}\right)(i=1,2, \cdots, g)$ may have an order of somewhat increasing complexity, that is, the simpler, the earlier.

EXAMPLE 5.1. A genealogical classification of the set $K(3,3)$ of all the configurations in a $3 \times 3$ cell space uinder LMT.

(a) The set of all the stable configurations $K^{(16)}(3,3)$. The lexicographical listing procedure given in Section 2 yields us the 16 stable configurations

$$
\begin{aligned}
& \left(\begin{array}{lll}
0 & 0 & 0 \\
0 & 0 & 0 \\
0 & 0 & 0
\end{array}\right)\left(\begin{array}{lll}
0 & 0 & 1 \\
0 & 0 & 1 \\
0 & 0 & 1
\end{array}\right) \\
& \left(\begin{array}{lll}
0 & 0 & 0 \\
0 & 0 & 0 \\
1 & 1 & 1
\end{array}\right)\left(\begin{array}{lll}
0 & 0 & 1 \\
0 & 0 & 1 \\
1 & 1 & 0
\end{array}\right) \quad\left(\begin{array}{lll}
0 & 1 & 0 \\
0 & 1 & 0 \\
0 & 1 & 0
\end{array}\right)
\end{aligned}
$$

$$
\begin{aligned}
& \left(\begin{array}{lll}
0 & 0 & 0 \\
1 & 1 & 1 \\
0 & 0 & 0
\end{array}\right) \quad\left(\begin{array}{lll}
0 & 0 & 1 \\
1 & 1 & 0 \\
0 & 0 & 1
\end{array}\right) \quad\left(\begin{array}{lll}
0 & 1 & 0 \\
1 & 0 & 1 \\
0 & 1 & 0
\end{array}\right) \quad\left(\begin{array}{lll}
0 & 1 & 1 \\
1 & 0 & 0 \\
0 & 1 & 1
\end{array}\right) \\
& \left(\begin{array}{lll}
0 & 0 & 0 \\
1 & 1 & 1 \\
1 & 1 & 1
\end{array}\right) \quad\left(\begin{array}{lll}
0 & 0 & 1 \\
1 & 1 & 0 \\
1 & 1 & 0
\end{array}\right) \quad\left(\begin{array}{lll}
0 & 1 & 0 \\
1 & 0 & 1 \\
1 & 0 & 1
\end{array}\right) \quad\left(\begin{array}{lll}
0 & 1 & 1 \\
1 & 0 & 0 \\
1 & 0 & 0
\end{array}\right)
\end{aligned}
$$

and the set of the remaining 16 stable configurations is a conjugate set of (5.21). In combination with rotation group consideration the whole set of 32 stable configurations can be classified into the following: 
(5.22)
$\left(1^{\circ}\right) \Psi\left(\begin{array}{lll}0 & 0 & 0 \\ 0 & 0 & 0 \\ 0 & 0 & 0\end{array}\right)$
$\overline{\left(1^{\circ}\right)} \Psi\left(\begin{array}{lll}1 & 1 & 1 \\ 1 & 1 & 1 \\ 1 & 1 & 1\end{array}\right)$
$\left(2^{\circ}\right) \quad \Theta\left(\begin{array}{lll}0 & 0 & 1 \\ 0 & 0 & 1 \\ 0 & 0 & 1\end{array}\right)$
$\Theta\left(\begin{array}{lll}1 & 1 & 0 \\ 1 & 1 & 0 \\ 1 & 1 & 0\end{array}\right)$
(3॰) $\Phi\left(\begin{array}{lll}0 & 1 & 0 \\ 0 & 1 & 0 \\ 0 & 1 & 0\end{array}\right)$
$\overline{\left(3^{\circ}\right)} \Phi\left(\begin{array}{lll}1 & 0 & 1 \\ 1 & 0 & 1 \\ 1 & 0 & 1\end{array}\right)$
$\left(4^{\circ}\right) \quad \Theta\left(\begin{array}{lll}0 & 1 & 1 \\ 1 & 0 & 0 \\ 1 & 0 & 0\end{array}\right)$
$\overline{\left(4^{\circ}\right)}$
$\Theta\left(\begin{array}{lll}1 & 0 & 0 \\ 0 & 1 & 1 \\ 0 & 1 & 1\end{array}\right)$
$\left(5^{\circ}\right) \quad \Theta\left(\begin{array}{lll}0 & 1 & 0 \\ 0 & 1 & 0 \\ 1 & 0 & 1\end{array}\right)$
$\Theta\left(\begin{array}{lll}1 & 0 & 1 \\ 1 & 0 & 1 \\ 0 & 1 & 0\end{array}\right)$
$\left(6^{\circ}\right)$

$$
\Psi\left(\begin{array}{lll}
0 & 1 & 0 \\
1 & 0 & 1 \\
0 & 1 & 0
\end{array}\right)
$$
$\Psi\left(\begin{array}{lll}1 & 0 & 1 \\ 0 & 1 & 0 \\ 1 & 0 & 1\end{array}\right)$

Each $\Theta$ group has 4 configurations, each $\Phi$ group 2 configurations, while each $\Psi$ group just one configuration. Consequently (5.22) represents the set of all the possible stable configurations in a $3 \times 3$ cell space, whose total number is 32 .

(b) The set of all the isolated stable configurations $S_{0}(3,3)$. The observations

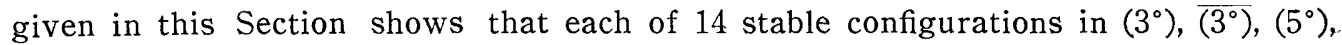
$\overline{\left(5^{\circ}\right)},\left(6^{\circ}\right)$ and $\overline{\left(6^{\circ}\right)}$ is isolated, and that the other $32-14=18$ stable configurations are non-isolated.

(c-1) Ancestor trees of $\left(4^{\circ}\right)$ and $\overline{\left(4^{\circ}\right)}$

$$
\mathfrak{N}\left(\Theta\left(\begin{array}{lll}
0 & 1 & 1 \\
1 & 0 & 0 \\
1 & 0 & 0
\end{array}\right)\right)=\Theta\left(\begin{array}{lll}
0 & 1 & 1 \\
1 & 1 & 0 \\
1 & 0 & 0
\end{array}\right)+\Theta\left(\begin{array}{lll}
0 & 1 & 1 \\
1 & 0 & 1 \\
1 & 0 & 0
\end{array}\right)+\Theta\left(\begin{array}{lll}
0 & 1 & 1 \\
1 & 0 & 0 \\
1 & 0 & 1
\end{array}\right),
$$

where each of the right-hand side has no direct ancestor. Consequently we have

$$
\begin{aligned}
\mathfrak{r}\left(\Theta\left(\begin{array}{lll}
0 & 1 & 1 \\
1 & 0 & 0 \\
1 & 0 & 0
\end{array}\right)\right) & =\sum_{\nu \geqq 0} a^{(\nu)}\left(\Theta\left(\begin{array}{lll}
0 & 1 & 1 \\
1 & 0 & 0 \\
1 & 0 & 0
\end{array}\right)\right) \\
& =\Theta\left(\begin{array}{lll}
0 & 1 & 1 \\
1 & 0 & 0 \\
1 & 0 & 0
\end{array}\right)+a\left(\Theta\left(\begin{array}{lll}
0 & 1 & 1 \\
1 & 0 & 0 \\
1 & 0 & 0
\end{array}\right)\right),
\end{aligned}
$$

as shown in Figure 5.2.

The ancestor tree of $\overline{\left(4^{\circ}\right)}$ can be obtained as the conjugates of (5.23) and (5.24). 


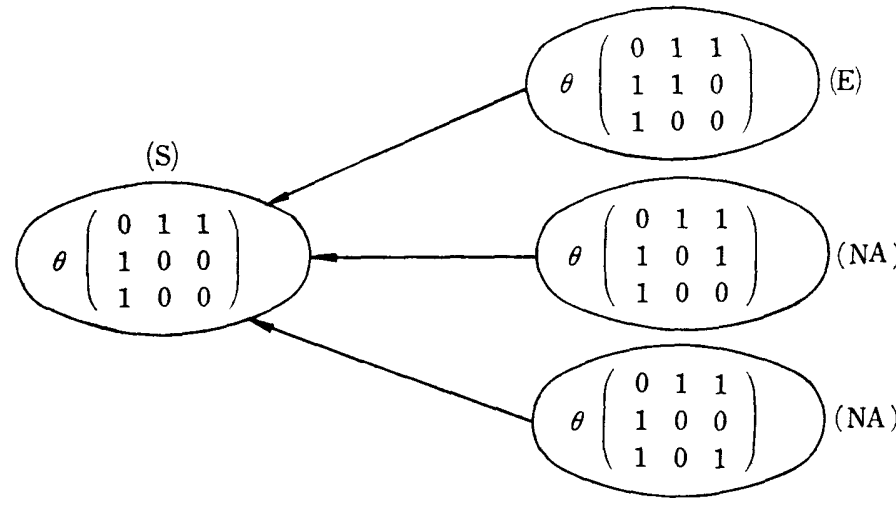

Fig. 5.2. Ancestor tree $\mathfrak{A} \Theta\left(\begin{array}{lll}0 & 1 & 1 \\ 1 & 0 & 0 \\ 1 & 0 & 0\end{array}\right)$

$(S)=$ stable configuration

$(E)=$ a configuration belonging to the Eden generation $(N A)=$ a configuration without ancestor.

In what follows we shall make use of the abbreviated notation

$$
a \Theta\left(\begin{array}{lll}
a_{11} & a_{12} & a_{13} \\
a_{21} & a_{22} & a_{23} \\
a_{31} & a_{32} & a_{33}
\end{array}\right)=a\left(\Theta\left(\begin{array}{lll}
a_{11} & a_{12} & a_{13} \\
a_{21} & a_{22} & a_{23} \\
a_{31} & a_{32} & a_{33}
\end{array}\right)\right)
$$

and similar ones $d \Theta(\cdot), \mathfrak{A} \Theta(\cdot)$ and $\mathfrak{D} \Theta(\cdot)$, for the sake of brevity.

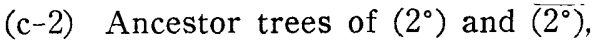

$$
a \Theta\left(\begin{array}{lll}
0 & 0 & 1 \\
0 & 0 & 1 \\
0 & 0 & 1
\end{array}\right)=\Theta\left(\begin{array}{lll}
1 & 0 & 1 \\
0 & 0 & 1 \\
0 & 0 & 1
\end{array}\right)+\Theta\left(\begin{array}{lll}
0 & 1 & 1 \\
0 & 0 & 1 \\
0 & 0 & 1
\end{array}\right)+\Theta\left(\begin{array}{lll}
0 & 0 & 1 \\
1 & 0 & 1 \\
0 & 0 & 1
\end{array}\right)+\Theta\left(\begin{array}{lll}
0 & 0 & 1 \\
0 & 1 & 1 \\
0 & 0 & 1
\end{array}\right)
$$

where the third and the fourth summands in the right-hand side have no direct ancestor. Regardirig the first and the second summands we have

$$
\begin{aligned}
& a \Theta\left(\begin{array}{lll}
1 & 0 & 1 \\
0 & 0 & 1 \\
0 & 0 & 1
\end{array}\right)=\Theta\left(\begin{array}{lll}
1 & 0 & 1 \\
1 & 0 & 1 \\
0 & 0 & 1
\end{array}\right)+\Theta\left(\begin{array}{lll}
1 & 0 & 1 \\
0 & 1 & 1 \\
0 & 0 & 1
\end{array}\right)+\Theta\left(\begin{array}{lll}
1 & 0 & 1 \\
0 & 0 & 1 \\
1 & 0 & 1
\end{array}\right)+\Theta\left(\begin{array}{lll}
1 & 0 & 1 \\
0 & 0 & 1 \\
0 & 1 & 1
\end{array}\right) \\
& a \Theta\left(\begin{array}{lll}
0 & 1 & 1 \\
0 & 0 & 1 \\
0 & 0 & 1
\end{array}\right)=\Theta\left(\begin{array}{lll}
0 & 1 & 1 \\
1 & 0 & 1 \\
0 & 0 & 1
\end{array}\right)+\Theta\left(\begin{array}{lll}
0 & 1 & 1 \\
0 & 1 & 1 \\
0 & 0 & 1
\end{array}\right)+\Theta\left(\begin{array}{lll}
0 & 1 & 1 \\
0 & 0 & 1 \\
1 & 0 & 1
\end{array}\right)+\Theta\left(\begin{array}{lll}
0 & 1 & 1 \\
0 & 0 & 1 \\
0 & 1 & 1
\end{array}\right)
\end{aligned}
$$

Now each of all the summands of the right-hand side in (5.27) and (5.28) has no direct ancestor: some of them belong to the Eden generation. In the consequence we have

$$
\mathfrak{H} \Theta\left(\begin{array}{lll}
0 & 0 & 1 \\
0 & 0 & 1 \\
0 & 0 & 1
\end{array}\right)=\sum_{\nu=0}^{2} a^{(\nu)} \Theta\left(\begin{array}{lll}
0 & 0 & 1 \\
0 & 0 & 1 \\
0 & 0 & 1
\end{array}\right)
$$




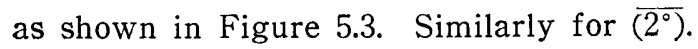

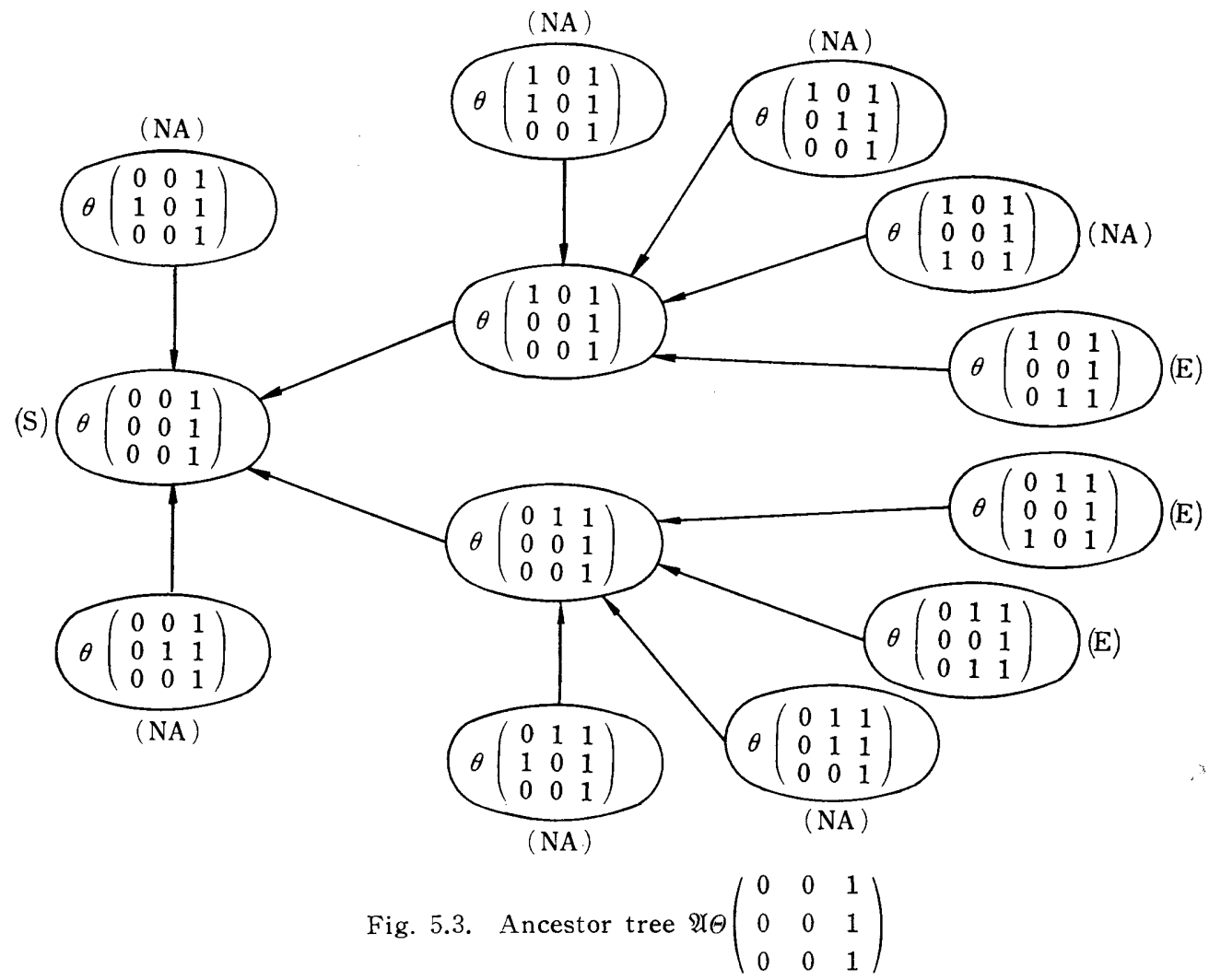

(c-3) Ancestor trees of $\left(1^{\circ}\right)$ and $\overline{\left(1^{\circ}\right)}$

$$
a \Theta\left(\begin{array}{lll}
0 & 0 & 0 \\
0 & 0 & 0 \\
0 & 0 & 0
\end{array}\right)=\Theta\left(\begin{array}{lll}
0 & 0 & 0 \\
0 & 0 & 1 \\
0 & 0 & 0
\end{array}\right)+\Theta\left(\begin{array}{lll}
0 & 0 & 1 \\
0 & 0 & 0 \\
0 & 0 & 0
\end{array}\right)+\Psi\left(\begin{array}{lll}
0 & 0 & 0 \\
0 & 1 & 0 \\
0 & 0 & 0
\end{array}\right) .
$$

In the right-hand side of (5.30), the third term belongs to the Eden generation, while the applications of observations mentioned in this Section yield us

$$
\begin{aligned}
a \Theta\left(\begin{array}{lll}
0 & 0 & 0 \\
0 & 0 & 1 \\
0 & 0 & 0
\end{array}\right) & =\Theta\left(\begin{array}{lll}
1 & 0 & 0 \\
0 & 0 & 1 \\
0 & 0 & 0
\end{array}\right)+\Theta\left(\begin{array}{lll}
0 & 1 & 0 \\
0 & 0 & 1 \\
0 & 0 & 0
\end{array}\right)+\Theta\left(\begin{array}{lll}
0 & 0 & 0 \\
1 & 0 & 1 \\
0 & 0 & 0
\end{array}\right) \\
& +\Theta\left(\begin{array}{lll}
0 & 0 & 0 \\
0 & 1 & 1 \\
0 & 0 & 0
\end{array}\right)+\Theta\left(\begin{array}{lll}
0 & 0 & 0 \\
0 & 0 & 1 \\
1 & 0 & 0
\end{array}\right)+\Theta\left(\begin{array}{lll}
0 & 0 & 0 \\
0 & 0 & 1 \\
0 & 1 & 0
\end{array}\right)
\end{aligned}
$$

In the right-hand side of (5.31), the third term belongs to the Eden generation and the fourth one has no ancestor, and we have also 


$$
a \Theta\left(\begin{array}{lll}
0 & 0 & 1 \\
0 & 0 & 1 \\
1 & 0 & 0
\end{array}\right)=\Theta\left(\begin{array}{lll}
1 & 0 & 1 \\
0 & 0 & 1 \\
1 & 0 & 0
\end{array}\right)+\Theta\left(\begin{array}{lll}
0 & 1 & 1 \\
0 & 0 & 1 \\
1 & 0 & 0
\end{array}\right)+\Theta\left(\begin{array}{lll}
0 & 0 & 1 \\
1 & 0 & 1 \\
1 & 0 & 0
\end{array}\right)+\Theta\left(\begin{array}{lll}
0 & 0 & 1 \\
0 & 1 & 1 \\
1 & 0 & 0
\end{array}\right)
$$

$$
\begin{aligned}
& a \Theta\left(\begin{array}{lll}
0 & 1 & 0 \\
0 & 0 & 1 \\
0 & 0 & 0
\end{array}\right)=\Theta\left(\begin{array}{lll}
0 & 1 & 0 \\
1 & 0 & 1 \\
0 & 0 & 0
\end{array}\right)+\Theta\left(\begin{array}{lll}
0 & 1 & 0 \\
0 & 1 & 1 \\
0 & 0 & 0
\end{array}\right)+\Theta\left(\begin{array}{lll}
0 & 1 & 0 \\
0 & 0 & 1 \\
1 & 0 & 0
\end{array}\right)+\Theta\left(\begin{array}{lll}
0 & 1 & 0 \\
0 & 0 & 1 \\
0 & 1 & 0
\end{array}\right) \\
& (N A) \\
& \text { (NA) }
\end{aligned}
$$

It is now convenient to introduce a set of configurations defined by

$$
\Theta_{1}\left(\begin{array}{lll}
a_{11} & a_{12} & a_{13} \\
a_{21} & a_{22} & a_{23} \\
a_{31} & a_{32} & a_{33}
\end{array}\right) \risingdotseq \Theta\left(\begin{array}{lll}
a_{11} & a_{12} & a_{13} \\
a_{21} & a_{22} & a_{23} \\
a_{31} & a_{32} & a_{33}
\end{array}\right)+\Theta\left(\begin{array}{lll}
a_{21} & a_{11} & a_{12} \\
a_{31} & a_{22} & a_{13} \\
a_{32} & a_{33} & a_{23}
\end{array}\right)
$$

Then the ancestor tree of the first term of the right-hand side of (5.30) can be shown as in Figure 5.4.

We summarize the result:

$$
\begin{aligned}
\mathfrak{H} \Theta\left(\begin{array}{lll}
0 & 0 & 0 \\
0 & 0 & 1 \\
0 & 0 & 0
\end{array}\right)= & \sum_{\nu=0}^{2} a^{(\nu)} \Theta\left(\begin{array}{lll}
0 & 0 & 0 \\
0 & 0 & 1 \\
0 & 0 & 0
\end{array}\right) \\
= & \Theta\left(\begin{array}{lll}
0 & 0 & 0 \\
0 & 0 & 1 \\
0 & 0 & 0
\end{array}\right)+\Theta\left(\begin{array}{lll}
0 & 0 & 0 \\
1 & 0 & 1 \\
0 & 0 & 0
\end{array}\right)+\Theta\left(\begin{array}{lll}
0 & 0 & 0 \\
0 & 1 & 1 \\
0 & 0 & 0
\end{array}\right) \\
& +\Theta_{1}\left(\begin{array}{lll}
0 & 0 & 1 \\
0 & 0 & 1 \\
1 & 0 & 0
\end{array}\right)+\Theta_{1}\left(\begin{array}{lll}
0 & 1 & 0 \\
0 & 0 & 1 \\
0 & 0 & 0
\end{array}\right) \\
& +\Theta_{1}\left(\begin{array}{lll}
1 & 0 & 1 \\
0 & 0 & 1 \\
1 & 0 & 0
\end{array}\right)+\Theta_{1}\left(\begin{array}{lll}
0 & 1 & 1 \\
0 & 1 & 1 \\
1 & 0 & 0
\end{array}\right)+\Theta_{1}\left(\begin{array}{lll}
0 & 0 & 1 \\
1 & 0 & 1 \\
1 & 0 & 0
\end{array}\right) \\
& +\Theta_{1}\left(\begin{array}{lll}
0 & 0 & 1 \\
0 & 1 & 1 \\
1 & 0 & 0
\end{array}\right)+\Theta_{1}\left(\begin{array}{lll}
0 & 1 & 0 \\
1 & 0 & 1 \\
0 & 0 & 0
\end{array}\right)+\Theta_{1}\left(\begin{array}{lll}
0 & 1 & 0 \\
0 & 1 & 1 \\
0 & 0 & 0
\end{array}\right) \\
& +\Theta_{1}\left(\begin{array}{lll}
0 & 1 & 0 \\
0 & 0 & 1 \\
1 & 0 & 0
\end{array}\right)+\Theta_{1}\left(\begin{array}{lll}
0 & 1 & 0 \\
0 & 0 & 1 \\
0 & 1 & 0
\end{array}\right) .
\end{aligned}
$$




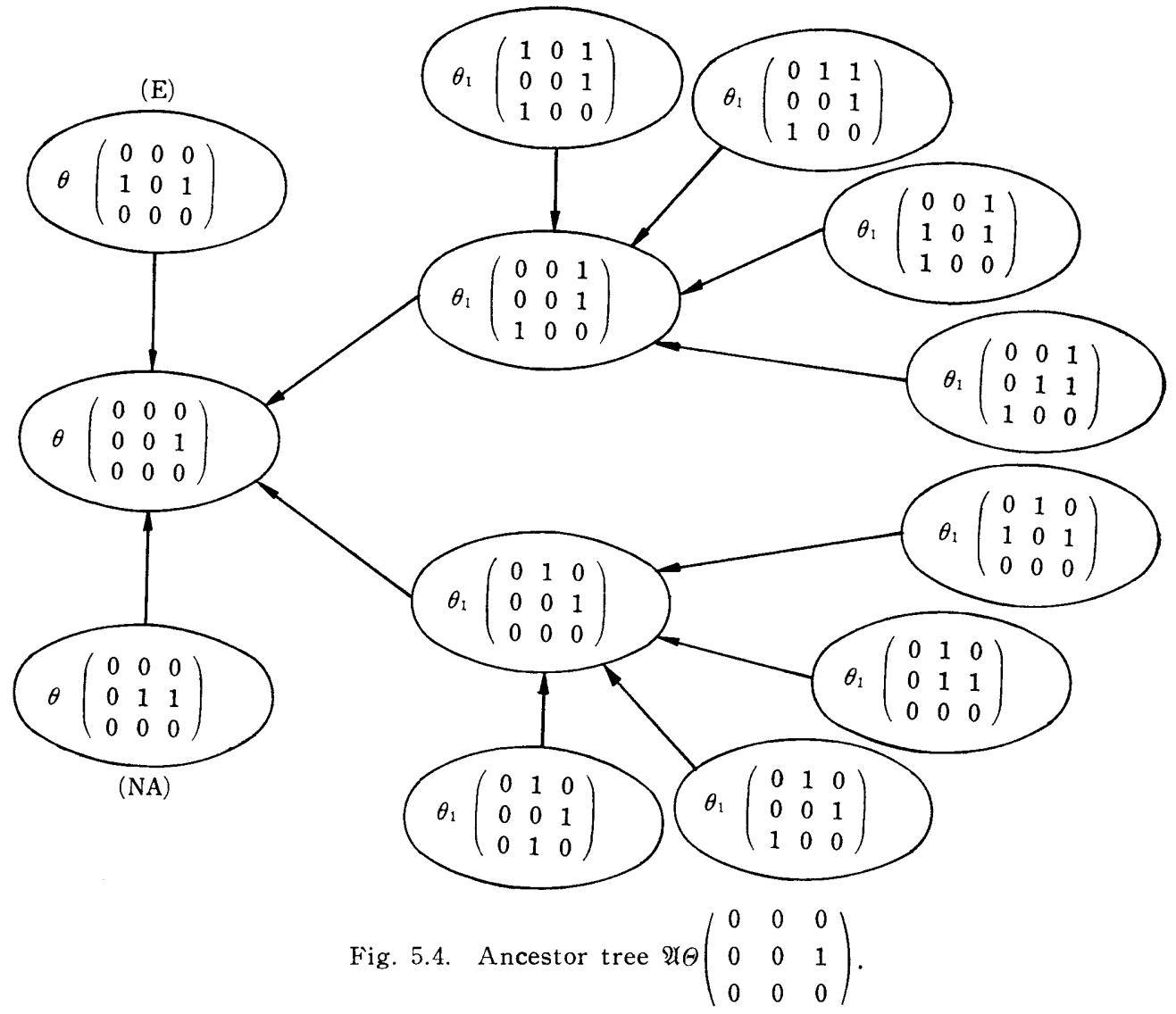

We have

$$
\begin{aligned}
& a \Theta\left(\begin{array}{lll}
0 & 0 & 1 \\
0 & 0 & 0 \\
0 & 0 & 0
\end{array}\right) \\
= & \Theta\left(\begin{array}{lll}
1 & 0 & 1 \\
0 & 0 & 0 \\
0 & 0 & 0
\end{array}\right)+\Theta\left(\begin{array}{lll}
0 & 1 & 1 \\
0 & 0 & 0 \\
0 & 0 & 0
\end{array}\right)+\Theta\left(\begin{array}{lll}
0 & 0 & 1 \\
1 & 0 & 0 \\
0 & 0 & 0
\end{array}\right)+\Theta\left(\begin{array}{lll}
0 & 0 & 1 \\
0 & 1 & 0 \\
0 & 0 & 0
\end{array}\right) \\
& +\Theta\left(\begin{array}{lll}
0 & 0 & 1 \\
0 & 0 & 1 \\
0 & 0 & 0
\end{array}\right)+\Theta\left(\begin{array}{lll}
0 & 0 & 1 \\
0 & 0 & 0 \\
1 & 0 & 0
\end{array}\right)+\Theta\left(\begin{array}{lll}
0 & 0 & 1 \\
0 & 0 & 0 \\
0 & 1 & 0
\end{array}\right)+\Theta\left(\begin{array}{lll}
0 & 0 & 1 \\
0 & 0 & 0 \\
0 & 0 & 1
\end{array}\right) \\
= & \Theta\left(\begin{array}{lll}
1 & 0 & 1 \\
0 & 0 & 0 \\
0 & 0 & 0
\end{array}\right)+\Theta_{1}\left(\begin{array}{lll}
1 & 0 & 1 \\
0 & 0 & 0 \\
0 & 0 & 0
\end{array}\right)+\Theta_{1}\left(\begin{array}{lll}
0 & 0 & 1 \\
1 & 0 & 0 \\
0 & 0 & 0
\end{array}\right)+\Theta\left(\begin{array}{lll}
0 & 0 & 1 \\
0 & 0 & 0 \\
1 & 0 & 0
\end{array}\right)+\Theta\left(\begin{array}{lll}
0 & 0 & 1 \\
0 & 1 & 0 \\
0 & 0 & 0
\end{array}\right),
\end{aligned}
$$

where the fifth term of the right hand side has no direct ancestor. We can proceed to find direct ancestors of the right-hand side quite similarly as before. Here we shall be content with giving the ancestor tree of the second summand 
$(5.37)$

$$
\begin{aligned}
a \Theta\left(\begin{array}{lll}
0 & 0 & 1 \\
0 & 0 & 1 \\
0 & 0 & 0
\end{array}\right)= & \Theta_{1}\left(\begin{array}{lll}
1 & 0 & 1 \\
0 & 0 & 1 \\
0 & 0 & 0
\end{array}\right)+\Theta_{1}\left(\begin{array}{lll}
0 & 1 & 1 \\
0 & 0 & 1 \\
0 & 0 & 0
\end{array}\right)+\Theta_{1}\left(\begin{array}{lll}
0 & 0 & 1 \\
1 & 0 & 1 \\
0 & 0 & 0
\end{array}\right) \\
& +\Theta_{1}\left(\begin{array}{lll}
0 & 0 & 1 \\
0 & 1 & 1 \\
0 & 0 & 0
\end{array}\right)+\Theta_{1}\left(\begin{array}{lll}
0 & 0 & 1 \\
0 & 0 & 1 \\
1 & 0 & 0
\end{array}\right)+\Theta_{1}\left(\begin{array}{lll}
0 & 0 & 1 \\
0 & 0 & 1 \\
0 & 1 & 0
\end{array}\right)
\end{aligned}
$$

In the right hand side of (5.37) we have $a+c+e+f+b+d$, say.

$$
\begin{aligned}
a \Theta_{1}\left(\begin{array}{lll}
1 & 0 & 1 \\
0 & 0 & 1 \\
0 & 0 & 0
\end{array}\right) & =\Theta\left(\begin{array}{lll}
1 & 0 & 1 \\
1 & 0 & 1 \\
0 & 0 & 0
\end{array}\right)+\Theta_{1}\left(\begin{array}{lll}
1 & 0 & 1 \\
0 & 1 & 1 \\
0 & 0 & 0
\end{array}\right)+\Theta_{1}\left(\begin{array}{lll}
1 & 0 & 1 \\
0 & 0 & 1 \\
1 & 0 & 0
\end{array}\right)+\Theta_{1}\left(\begin{array}{lll}
1 & 0 & 1 \\
0 & 0 & 1 \\
0 & 1 & 0
\end{array}\right) \\
& =a_{1}+a_{2}+a_{3}+a_{4} \text {, say }
\end{aligned}
$$

$$
\begin{aligned}
a \Theta_{1}\left(\begin{array}{lll}
0 & 0 & 1 \\
0 & 0 & 1 \\
1 & 0 & 0
\end{array}\right) & =\Theta_{1}\left(\begin{array}{lll}
1 & 0 & 1 \\
0 & 0 & 1 \\
1 & 0 & 0
\end{array}\right)+\Theta_{1}\left(\begin{array}{lll}
0 & 1 & 1 \\
0 & 0 & 1 \\
1 & 0 & 0
\end{array}\right)+\Theta_{1}\left(\begin{array}{lll}
1 & 0 & 1 \\
1 & 0 & 1 \\
1 & 0 & 0
\end{array}\right)+\Theta_{1}\left(\begin{array}{lll}
0 & 0 & 1 \\
0 & 1 & 1 \\
1 & 0 & 0
\end{array}\right) \\
& =b_{1}+b_{2}+b_{3}+b_{4} \text {, say }
\end{aligned}
$$

$$
\begin{aligned}
a \Theta_{1}\left(\begin{array}{lll}
0 & 1 & 1 \\
0 & 0 & 1 \\
0 & 0 & 0
\end{array}\right) & =\Theta_{1}\left(\begin{array}{lll}
0 & 1 & 1 \\
1 & 0 & 1 \\
0 & 0 & 0
\end{array}\right)+\Theta_{1}\left(\begin{array}{lll}
0 & 1 & 1 \\
0 & 1 & 1 \\
0 & 0 & 0
\end{array}\right)+\Theta_{1}\left(\begin{array}{lll}
0 & 1 & 1 \\
0 & 0 & 1 \\
1 & 0 & 0
\end{array}\right)+\Theta_{1}\left(\begin{array}{lll}
0 & 1 & 1 \\
0 & 0 & 1 \\
0 & 1 & 0
\end{array}\right) \\
& =c_{1}+c_{2}+c_{3}+c_{4} \text {, say }
\end{aligned}
$$

$$
\begin{aligned}
a \Theta_{1}\left(\begin{array}{lll}
0 & 1 & 1 \\
0 & 1 & 1 \\
0 & 0 & 0
\end{array}\right) & =\Theta_{1}\left(\begin{array}{lll}
0 & 0 & 1 \\
0 & 1 & 1 \\
0 & 0 & 0
\end{array}\right)+\Theta_{1}\left(\begin{array}{lll}
0 & 1 & 0 \\
0 & 1 & 1 \\
0 & 0 & 0
\end{array}\right)+\Theta_{1}\left(\begin{array}{lll}
0 & 1 & 1 \\
0 & 0 & 1 \\
0 & 0 & 0
\end{array}\right)+\Theta_{1}\left(\begin{array}{lll}
0 & 1 & 1 \\
0 & 1 & 0 \\
0 & 0 & 0
\end{array}\right) \\
& =c_{2,1}+c_{2,2}+c_{2,3}+c_{2,4} \text {, say }
\end{aligned}
$$

$$
\begin{aligned}
a \Theta_{1}\left(\begin{array}{lll}
0 & 0 & 1 \\
0 & 0 & 1 \\
0 & 1 & 0
\end{array}\right) & =\Theta_{1}\left(\begin{array}{lll}
1 & 0 & 1 \\
0 & 0 & 1 \\
0 & 1 & 0
\end{array}\right)+\Theta_{1}\left(\begin{array}{lll}
0 & 1 & 1 \\
0 & 0 & 1 \\
0 & 1 & 0
\end{array}\right)+\Theta_{1}\left(\begin{array}{lll}
0 & 0 & 1 \\
1 & 0 & 1 \\
0 & 1 & 0
\end{array}\right)+\Theta_{1}\left(\begin{array}{lll}
0 & 0 & 1 \\
0 & 1 & 1 \\
0 & 1 & 0
\end{array}\right) \\
& =d_{1}+d_{2}+d_{3}+d_{4} \text {, say. }
\end{aligned}
$$

Here we notice that $e, f, a_{i}, b_{i}, c_{2,1}, c_{2,3}, c_{2,4}, d_{1}, d_{2}, d_{3}$ and $d_{4}$ has no ancestor, in fact some of them belong to Eden generation and that $a_{3}=b_{1}, b_{2}=c_{3}, c_{4}=d_{1}, d_{1}=a_{4}$.

The considerations given just now are sufficient to construct an ancestor tree for (5.37), as shown in Figure 5.5.

(d) The lineage

There are no novelities in finding out descendant trees. The completion of the whole ancestor and descendant relations among all the configurations would be laborous and tedious even in this simple Example. In order to observe the lineage relations among all the configurations in a $3 \times 3$ cell space, we have to be concerned with the problem how many components do there exist. So far as this problem is concerned, our answer is given by Figure 5.6 in which a route connecting all the 


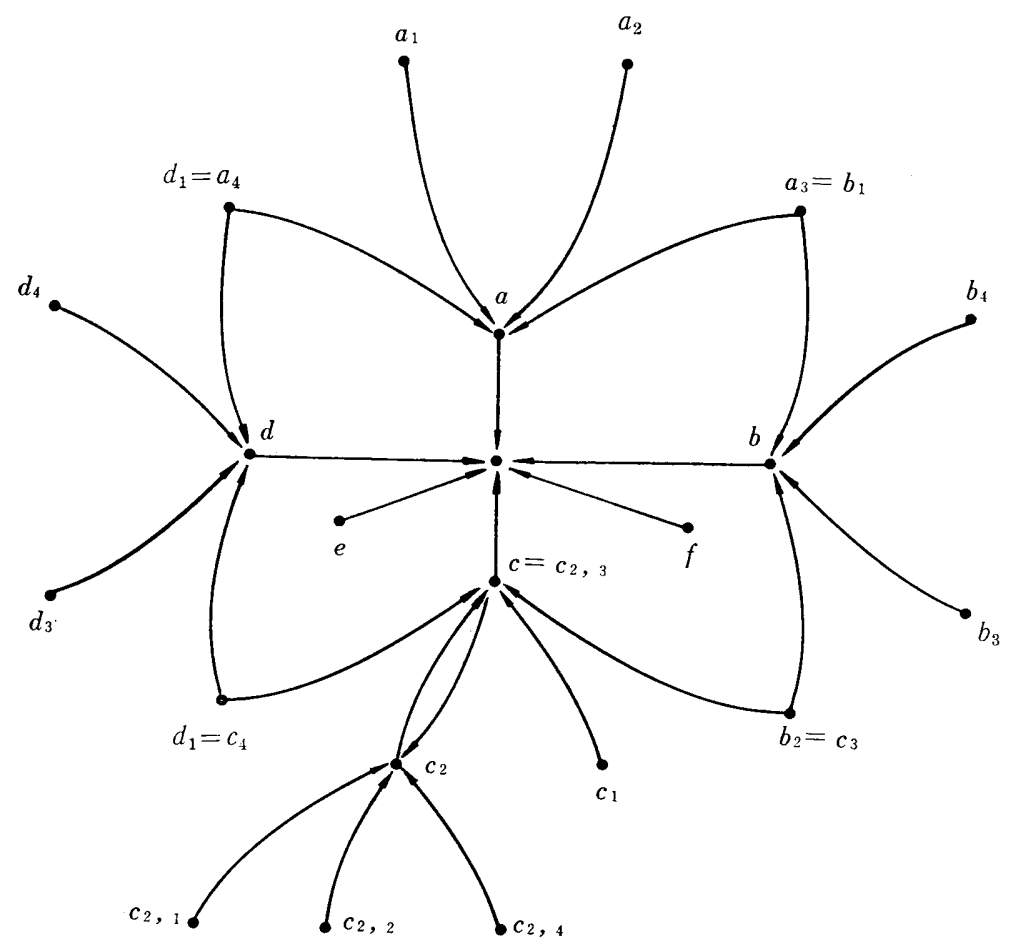

Fig. 5.5. The ancestor tree

$$
\Theta\left(\begin{array}{lll}
0 & 0 & 1 \\
0 & 0 & 1 \\
0 & 0 & 0
\end{array}\right) .
$$

non-isolated stable configurations $\left(1^{\circ}\right), \overline{\left(1^{\circ}\right)},\left(2^{\circ}\right),\left(2^{\circ}\right),\left(4^{\circ}\right)$ and $\overline{\left(4^{\circ}\right)}$ in $(5.22)$ are shown, where the notion of $\Theta$ rotations is not described for the same of brevity. This implies that all the non-isolated stable configurations constitute one lineage. It is evident that each isolated stable configuration has no ancestor and no descendant different from itself and hence constitute a (singular) lineage respectively.

Now we proceed to prove

THEOREM II. The set of all the configurations in an $m \times n$ cell space, for $m, n \geqq 3$, which are not isolated stable configurations constitutes one lineage. Each individual isolated stable configuration constitutes one lineage consisted of itself.

In order to prove this Theorem, we prapare a few Lemmas which have some interests in themselves. In a general pattern of all the stable configurations in an $m \times n$ cell space given in THEOREM 3 in Kitagawa-Yamaguchi [1], let us define

$$
\begin{aligned}
& m_{0}=\operatorname{Max}_{1 \leqq i \leqq k}\left\{m_{i}\right\} . \\
& n_{0}=\operatorname{Max}_{1 \leqq i \leqq l}\left\{n_{j}\right\} .
\end{aligned}
$$

In what follows we shall be concerned with the cases when $m, n \geqq 3$, because other cases have been either substantially discussed in our previous Examples or 


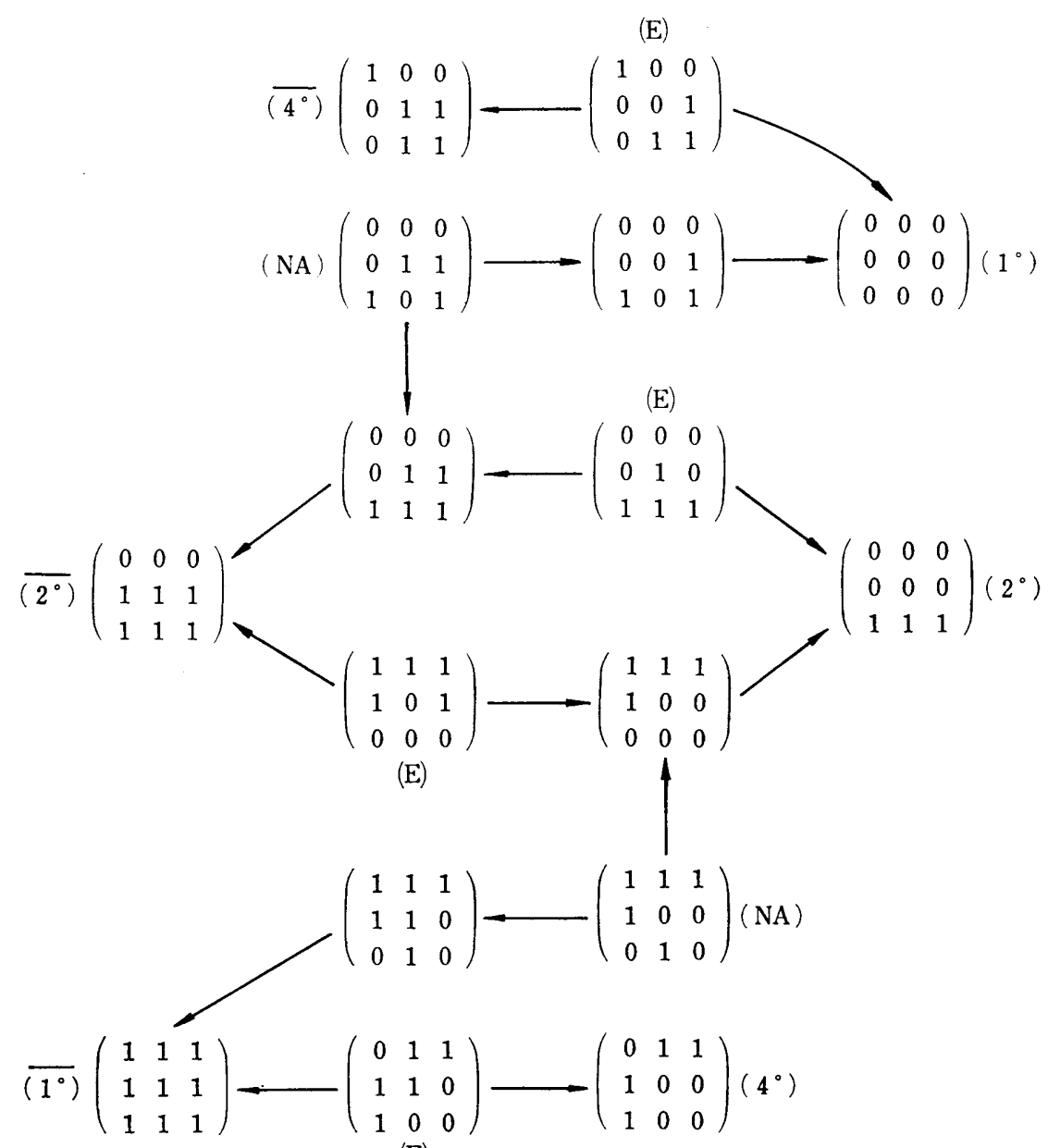

(E)

Fig. 5.6. A lineage route connecting all the non-isolated stable configurations $\left(1^{\circ}\right), \overline{\left(1^{\circ}\right)},\left(2^{\circ}\right), \overline{\left(2^{\circ}\right)},\left(4^{\circ}\right)$ and $\overline{\left(4^{\circ}\right)}$ in $(5.22)$.

can be deduced from what we will discuss.

LEMMA 2. A stable configuration is isolated if and only if it satisfies either one of the following three conditions
(a) $m_{0}=n_{0}=1$
(b) $\quad m>m_{0} \geqq 2$ and $n_{0}=1$
(c) $\quad m_{0}=1$ and $n>n_{0} \geqq 2$.

The sufficiency of (5.45) is immediate, while the necessity follows from the combination of the following LEMMAS 3 and 4 which deal with all the other remaining cases and which prove the non-isolation of those stable configurations.

LEMMA 3. Each stable configuration which satisfies the condition

(d) $\quad m>m_{0} \geqq 2$ and $n_{0}>n \geqq 2$ 
has an ancestor common with at least either of the two configurations $C_{0}$ and $C_{1}$.

PROOF. Let us consider the case (d). Due to the condition (5.46), (d), a stable configuration has at least one $3 \times 3$ cell space of the following types
(i) $\left(\begin{array}{lll}0 & 1 & 1 \\ 1 & 0 * & 0 \\ 1 & 0 & 0\end{array}\right)$
(ii) $\left(\begin{array}{lll}1 & 0 & 0 \\ 1 & 0 * & 0 \\ 0 & 1 & 1\end{array}\right)$
(iii) $\left(\begin{array}{lll}0 & 0 & 1 \\ 0 & 0 * & 1 \\ 1 & 1 & 0\end{array}\right)$
(iv) $\left(\begin{array}{lll}1 & 1 & 0 \\ 0 & 0 * & 1 \\ 0 & 0 & 1\end{array}\right)$
(v) $\left(\begin{array}{lll}1 & 0 & 0 \\ 0 & 1 * & 1 \\ 0 & 1 & 1\end{array}\right)$
(vi) $\left(\begin{array}{lll}0 & 1 & 1 \\ 0 & 1 * & 1 \\ 1 & 0 & 0\end{array}\right)$
(vii) $\left(\begin{array}{lll}1 & 1 & 0 \\ 1 & 1 * & 0 \\ 0 & 0 & 1\end{array}\right)$
(viii) $\left(\begin{array}{lll}0 & 0 & 1 \\ 1 & 1^{*} & 0 \\ 1 & 1 & 0\end{array}\right)$

By changing $0^{*}$ and $1^{*}$ by 1 and 0 respectively we get a $2 \times 2$ basic cell space which, in combination of the other unchanged configuration, becomes to be a direct ancestor of the given configuration as well as to be an ancestor of $C_{1}$ and $C_{0}$ respectively. This completes the proof of the first part of the assertion of LEMMA 3 . The second part is verified by the existence of stable configurations having both replacements of 1 and 0 by $0^{*}$ and $1^{*}$ respectively.

LEMMA 4. Each stable configuration which satisfies either of the following two conditions

$$
\begin{aligned}
& \text { (e) } \quad m=m_{0} \text { and } n>n_{0} \geqq 2 \\
& \text { (f) } m>m_{0} \geqq 2 \text { and } n=n_{0}
\end{aligned}
$$

has an ancestor common to the configuration $C_{0}$ and also that common to the configuration $C_{1}$.

The proof is given only for the case (e), because the case (f) can be treated quite similarly. In order to simplify our descriptions, let us introduce several kinds of $m$-dimensional vectors

$$
\begin{aligned}
1_{\omega}=\left(\begin{array}{c}
1 \\
1 \\
1 \\
\vdots \\
1
\end{array}\right), & 0_{\omega}=\left(\begin{array}{c}
0 \\
0 \\
0 \\
\vdots \\
0
\end{array}\right) \\
1_{\alpha}=\left(\begin{array}{c}
0 \\
0 \\
\vdots \\
0 \\
1 \\
0 \\
\vdots \\
0
\end{array}\right), & 0_{\alpha}=\left(\begin{array}{c}
1 \\
1 \\
\vdots \\
1 \\
0 \\
1 \\
\vdots \\
1
\end{array}\right),
\end{aligned}
$$

which means that $1_{\alpha}\left(0_{\alpha}\right)$ has the sole one (zero) at some (suitably chosen) component. By means of the combination of these vectors we can denote a certain class of configurations.

For instance we have 


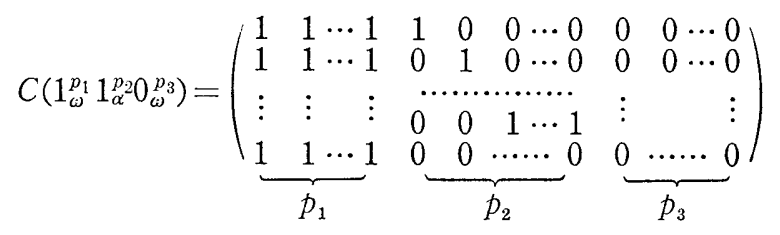

Now a stable configuration satisfying the condition (e) is either one of the following four configurations

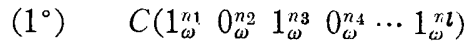

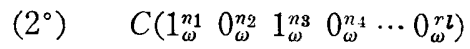

$$
\begin{aligned}
& \left(3^{\circ}\right) \quad C\left(\begin{array}{lllllll}
0_{\omega}^{n_{1}} & 1_{\omega}^{n_{2}} & 0_{\omega}^{n_{3}} & 1_{\omega}^{n_{4}} & \cdots & \left.1_{\omega}^{n^{n}}\right)
\end{array}\right) \\
& \left(4^{\circ}\right) \quad C\left(\begin{array}{lllllll}
0_{\omega}^{n_{1}} & 1_{\omega}^{n_{2}} & 0_{\omega}^{n_{3}} & 1_{\omega}^{n_{4}} & \cdots & \left.0_{\omega}^{n^{n}}\right)
\end{array}\right),
\end{aligned}
$$

for which $n_{0}=\max \left(n_{j}\right) \geqq 2$.

Furthermore two configuration $C^{(1)}$ and $C^{(2)}$ is denoted by $C^{(1)} \rightarrow C^{(2)}$ if there exists a LMT which transforms $C^{(1)}$ to $C^{(2)}$. For instance, we observe, in view of (5.51)

$$
\begin{aligned}
& C\left(1_{\omega}^{p_{1}} 1_{\alpha}^{p_{2}} 0_{\omega}^{p_{3}}\right) \longrightarrow C\left(1_{\omega}^{p_{1}} 1_{\alpha}^{p_{2}-1} 0_{\omega}^{p_{3}+1}\right) \\
& C\left(1_{\omega}^{p_{1}} 1_{\alpha}^{p_{2}} 0_{\omega}^{p_{3}}\right) \longrightarrow C\left(1_{\omega}^{p_{1}+1} 1_{\alpha}^{p_{2}-1} 0_{\omega}^{p_{3} 3}\right)
\end{aligned}
$$

and so on.

After these preparations, let us now turn to

Proof of LEMMA 4. There are $n$ sequences of applications of LMT such that

$$
\begin{aligned}
& C\left(1_{\omega} 1_{\alpha}^{n-1}\right) \longrightarrow C\left(1_{\omega}^{2} 1_{\alpha}^{n-2}\right) \longrightarrow C\left(1_{\omega}^{3} 1_{\alpha}^{n-3}\right) \longrightarrow \cdots \rightarrow C\left(1_{\omega}^{n}\right) \\
& C\left(1_{\omega}^{k} 1_{\alpha}^{n-k}\right) \longrightarrow C\left(1_{\omega}^{k} 1_{\alpha}^{n-k-1} 0_{\omega}\right) \longrightarrow \cdots \longrightarrow C\left(1_{\omega}^{k} 0_{\omega}^{n-k}\right)
\end{aligned}
$$

for $k=1,2, \cdots, n-1$. The combinations of these $n$ sequences shows that $C\left(1_{\omega} 1_{\alpha}^{n-1}\right)$ is a common ancestor to all the configurations in these $n$ sequences. In particular $C\left(1_{\omega}^{k} 0_{\omega}^{n-k}\right)(k=1,2, \cdots, n)$ belong to the same lineage which is denoted by

$$
\begin{aligned}
C\left(1_{\omega} 0_{\omega}^{n-1}\right) & \sim C\left(1_{\omega}^{2} 0_{\omega}^{n-2}\right) \sim C\left(1_{\omega}^{3} 0_{\omega}^{n-3}\right) \sim \cdots \\
& \sim C\left(1_{\omega}^{k} 0_{\omega}^{n-k}\right) \sim \cdots \sim C\left(1_{\omega}^{n-1} 0_{\omega}\right) \sim C\left(1_{\omega}^{n}\right)
\end{aligned}
$$

and more simply by

(i) $10^{n-1} \sim 1^{2} 0^{n-2} \sim 1^{3} 0^{n-3} \sim \cdots \sim 1^{k} 0^{n-k} \sim \cdots \sim 1^{n}$.

Similar argument gives us also

$$
\begin{aligned}
& \text { (ii) } 0^{n-1} 1 \sim 0^{n-2} 1^{2} \sim 0^{n-3} 1^{3} \sim \cdots \sim 0^{n-k} 1^{k} \sim \cdots \sim 1^{n} \\
& \text { (iii) } 1^{n-1} 0 \sim 1^{n-2} 0^{2} \sim 1^{n-3} 0^{3} \sim \cdots \sim 1^{n-k} 0^{k} \sim \cdots \sim 0^{n} \\
& \text { (iv) } 01^{n-1} \sim 0^{2} 1^{n-2} \sim 0^{3} 1^{n-3} \sim \cdots \sim 0^{k} 1^{n-k} \sim \cdots \sim 0^{n} .
\end{aligned}
$$

In combination for (i), (ii), (iii), (iv) in (5.58) we have 


$$
\begin{aligned}
& 0^{n} \sim 0^{n-1} 1 \sim 0^{n-2} 1^{2} \sim \cdots \sim 0^{2} 1^{n-2} \sim 01^{n-1} \sim 1^{n} \\
& 0^{n} \sim 10^{n-1} \sim 1^{2} 0^{n-2} \sim \cdots \sim 1^{n-2} 0^{2} \sim 1^{n-1} 0 \sim 1^{n},
\end{aligned}
$$

that is, the set of configurations $\left\{0^{n-k} 1^{k}, 1^{k} 0^{n-k}\right.$; for $\left.k=0,1,2, \cdots, n\right\}$ belongs to the same lineage.

The result shown in (5.59) can be applied to each case $\left(1^{\circ}\right),\left(2^{\circ}\right),\left(3^{\circ}\right)$ and $\left(4^{\circ}\right)$ in (5.52), since there exists at least one $n_{p}$ such that $n_{p}=n_{0} \geqq 2$, and through all cases we find that they belong to the same lineage with the configurations $C_{0}$ and $C_{1}$, as we were to prove.

It is noted that the non-isolated configurations $C_{0}$ and $C_{1}$ satisfy the condition

$$
\text { (g) } \quad m=m_{0}, \quad n=n_{0}
$$

which, in combination with (5.48) (a) (f), exhausts all the possible cases for the set of all the stable configurations in an $m \times n$ cell space. In the consequence we have

LEMMA 5. The set of all the non-isolated stable configurations constitutes one lineage.

This is evident from the combination of LEMMA 4 and 5, in view of (5.48)(d), (e), (f), and (g).

PROOF OF THEOREM II. To any assigned configuration, which is not an isolated stable configuration, in an $m \times n$ cell space, there exists a sequence of LMT whose applications lead to a non-isolated stable configuration, that is to say, it is an ancestor of at least one non-isolated stable configuration, and hence belongs to the same lineage with this non-isolated stable configuration. Since the set of all the nonisolated stable configurations constitute one lineage, any assigned configuration which is not an isolated stable configuration belongs to the same lineage, as we were to prove.

\section{References}

[1] Kitagawa, T. and Yamaguchi, M.: Local Majority Transformation in Cell Space, Information Science Approach to Biomathematics, II, Research Report of Research Institute of the Fundamental Science, Fac. Sci. Kyushu Univ., No. 10, 1970, and Bull. Math. Stat. Vol. 14, No. 3/4 (1971) 61-82.

[2] Yamaguchi, M.: The Stability Index of Stable Configurations under Local Majority Transformations on Cell Space-Information Science Approach to Biomathematice, III, Research Report of Research Institute of the Fundamental Science, Fac. Sci. Kyushu Univ., No. 11, 1970, and Bull. Math. Stat. Vol. 14, No. 3/4 (1971), 83-91.

[3] Kitagawa, T.: Prolegomena to Cell Space Approaches-Information Science Approach to Biomathematics, IV, Research Report of the Research Institute of the Fundamental Information Science, Fac. Sci. Kyushu Univ., and Mem. Fac. Sci., Kyushu Univ., Ser. A, 26 (1971), 1-73. 